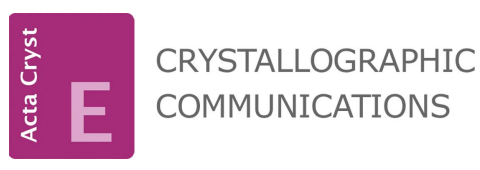

ISSN 2056-9890

Received 20 May 2019

Accepted 15 June 2019

Edited by H. Stoeckli-Evans, University of Neuchâtel, Switzerland

Keywords: crystal structure; pyridazin-3(2H)one; hydrogen bonding; $\mathrm{C}-\mathrm{H} \cdots \pi$ interaction.

CCDC reference: 1923216

Supporting information: this article has supporting information at journals.iucr.org/e

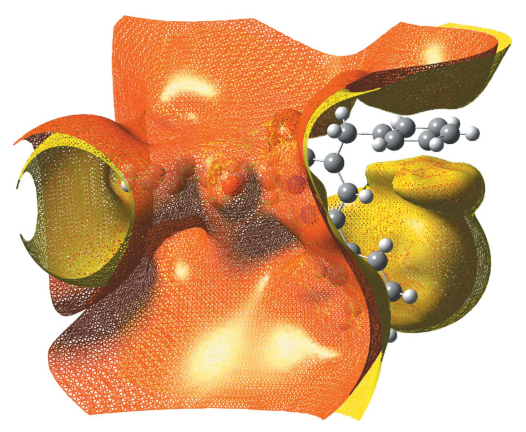

OPEN $\odot$ ACCESS

\section{Crystal structure and the DFT and MEP study of 4-benzyl-2-[2-(4-fluorophenyl)-2-oxoethyl]-6- phenylpyridazin-3(2H)-one}

\author{
Said Daoui, ${ }^{a *}$ Md. Serajul Haque Faizi, ${ }^{\text {b* }}$ Fouad El Kalai, ${ }^{a}$ Rafik Saddik, ${ }^{c}$ Necmi \\ Dege, ${ }^{d}$ Khalid Karrouchi $^{\mathrm{e}}$ and Noureddine Benchat ${ }^{\mathrm{a}}$
}

\author{
aLaboratory of Applied Chemistry and Environment (LCAE), Department of Chemistry, Faculty of Sciences, University \\ Mohamed Premier, Oujda 60000, Morocco, ${ }^{\mathbf{b}}$ Department of Chemistry, Langat Singh College, Babasaheb Bhimrao \\ Ambedkar Bihar University, Muzaffarpur, Bihar-842001, India, 'Laboratory of Organic Synthesis, Extraction and

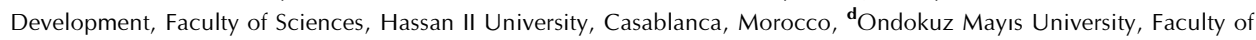 \\ Arts and Sciences, Department of Physics, 55139, Kurupelit, Samsun, Turkey, and 'Laboratory of Plant Chemistry, \\ Organic and Bioorganic Synthesis, URAC23, Faculty of Science, BP 1014, GEOPAC Research Center, Mohammed V \\ University, Rabat, Morocco. *Correspondence e-mail: saiddaoui26@gmail.com, faizichemiitg@gmail.com
}

The title pyridazin-3(2H)-one derivative, $\mathrm{C}_{25} \mathrm{H}_{19} \mathrm{FN}_{2} \mathrm{O}_{2}$, crystallizes with two independent molecules $(A$ and $B)$ in the asymmetric unit. In molecule $A$, the 4-fluorophenyl ring, the benzyl ring and the phenyl ring are inclined to the central pyridazine ring by $86.54(11), 3.70(9)$ and $84.857(13)^{\circ}$, respectively. In molecule $B$, the corresponding dihedral angles are 86.80 (9), 10.47 (8) and $82.01(10)^{\circ}$, respectively. In the crystal, the $A$ molecules are linked by pairs of $\mathrm{C}-\mathrm{H} \cdots \mathrm{F}$ hydrogen bonds, forming inversion dimers with an $R_{2}^{2}(28)$ ring motif. The dimers are linked by $\mathrm{C}-\mathrm{H} \cdots \mathrm{O}$ hydrogen bonds and a $\mathrm{C}-\mathrm{H} \cdots \pi$ interaction, forming columns stacking along the $a$-axis direction. The $B$ molecules are linked to each other in a similar manner and form columns separating the columns of $A$ molecules.

\section{Chemical context}

Pyridazin-3(2H)-ones are pyridazine derivatives, being constructed about a six-membered ring that contains two adjacent nitrogen atoms, at positions one and two, and with a carbonyl group at position three. The interest in these nitrogen-rich heterocyclic derivatives arises from the fact that they exhibit a number of promising pharmacological and biological activities. These include anti-oxidant (Khokra et al., 2016), anti-bacterial and anti-fungal (Abiha et al. 2018), anti-cancer (Kamble et al. 2017), analgesic and anti-inflammatory (Ibrahim et al. 2017), anti-depressant (Boukharsa et al. 2016) and anti-ulcer activities (Yamada et al., 1981). In addition, a number of pyridazinone derivatives have been reported to have potential as agrochemicals, for example as insecticides (Nauen \& Bretschneider, 2002), acaricides (Igarashi \& Sakamoto, 1994) and herbicides (Azaari et al., 2016). The present work is a part of an ongoing structural study of heterocyclic compounds and their utilization as molecular (Faizi et al., 2016) and fluorescence (Mukherjee et al., 2018; Kumar et al., 2017; 2018) sensors. Given the interest in this class of compounds and the paucity of structural data, the crystal structure analysis of the title pyridazin-3 $(2 H)$-one derivative has been undertaken, along with a DFT study, in order to gain further insight into the molecular structure. 
<smiles>O=C(Cn1nc(-c2ccccc2)cc(Cc2ccccc2)c1=O)c1ccc(F)cc1</smiles>

\section{Structural commentary}

The title compound crystallizes with two independent molecules $(A$ and $B)$ in the asymmetric unit (Fig. 1). In each molecule, a central oxopyridazinyl ring is connected to a fluorobenzylacetate group, a phenyl group, and a benzyl residue. The oxopyridazinyl ring $(B)$ is planar in both molecules; r.m.s. deviations are $0.029 \AA$ for molecule $A$ and $0.009 \AA$ for molecule $B$. In molecule $A$, the 4-fluorophenyl ring $(A ; \mathrm{C} 1 A-\mathrm{C} 6 A)$, the benzyl ring $(C ; \mathrm{C} 20 A-\mathrm{C} 25 A)$ and the phenyl ring $(D ; \mathrm{C} 13 A-\mathrm{C} 18 A)$ are inclined to the central pyridazine ring $(B ; \mathrm{N} 1 A / \mathrm{N} 2 A / \mathrm{C} 9 A-\mathrm{C} 12 A)$ by $86.54(11)$, $3.70(9)$ and $84.87(13)^{\circ}$, respectively. In molecule $B$, the corresponding dihedral angles are $86.80(9), 10.47(8)$ and $82.01(10)^{\circ}$, respectively. Hence, the conformation of the two molecules differs essentially in the orientation of the benzyl ring $(C)$ with respect to the central pyridazine ring $(B)$; $3.70(9)^{\circ}$ in molecule $A$ compared to $10.47(8)^{\circ}$ in molecule $B$. The two molecules have an r.m.s. deviation of $0.683 \AA$ for the 30 non-hydrogen atoms (Fig. 2; PLATON; Spek, 2009).

\section{Supramolecular features}

In the crystal, the $A$ molecules are linked by pairs of $\mathrm{C}-\mathrm{H} \cdots \mathrm{F}$ hydrogen bonds, forming inversion dimers with an $R_{2}^{2}(28)$ ring motif (Table 1 and Fig. 3). The dimers are linked by $\mathrm{C}-\mathrm{H} \cdots \mathrm{O}$ hydrogen bonds and a $\mathrm{C}-\mathrm{H} \cdots \pi$ interaction (Table 1), forming columns stacking along the $a$-axis direction. The $B$

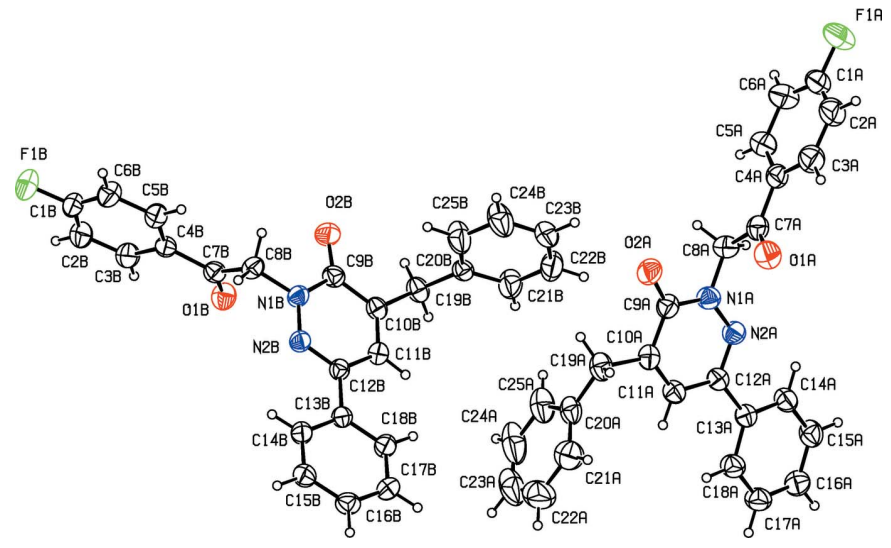

Figure 1

The molecular structure of the title compound, with the atom labelling and displacement ellipsoids drawn at the $30 \%$ probability level.
Table 1

Hydrogen-bond geometry $\left(\AA,^{\circ}\right)$.

$C g 1$ is the centroid of the $\mathrm{N} 1 A / \mathrm{N} 2 A / \mathrm{C} 9 A-\mathrm{C} 12 A$ ring.

\begin{tabular}{lllll}
\hline$D-\mathrm{H} \cdots A$ & $D-\mathrm{H}$ & $\mathrm{H} \cdots A$ & $D \cdots A$ & $D-\mathrm{H} \cdots A$ \\
\hline $\mathrm{C} 15 A-\mathrm{H} 15 A \cdots \mathrm{F} 1 A^{\mathrm{i}}$ & 0.93 & 2.49 & $3.263(3)$ & 141 \\
$\mathrm{C} 15 B-\mathrm{H} 15 B \cdots \mathrm{F} 1 B^{\mathrm{ii}}$ & 0.93 & 2.56 & $3.310(3)$ & 138 \\
$\mathrm{C} 8 A-\mathrm{H} 8 B \cdots \mathrm{O} 1 A^{\text {iii }}$ & 0.97 & 2.50 & $3.466(3)$ & 179 \\
$\mathrm{C} 8 B-\mathrm{H} 8 D \cdots \mathrm{O} 1 B^{\text {iv }}$ & 0.97 & 2.49 & $3.458(2)$ & 176 \\
$\mathrm{C} 19 A-\mathrm{H} 19 A \cdots C g 1^{\text {iv }}$ & 0.97 & 2.93 & $3.845(2)$ & 158 \\
\hline
\end{tabular}

Symmetry codes: (i) $-x+2,-y+1,-z$; (ii) $-x-1,-y,-z+1$; (iii) $x+1, y, z$; (iv) $x-1, y, z$.

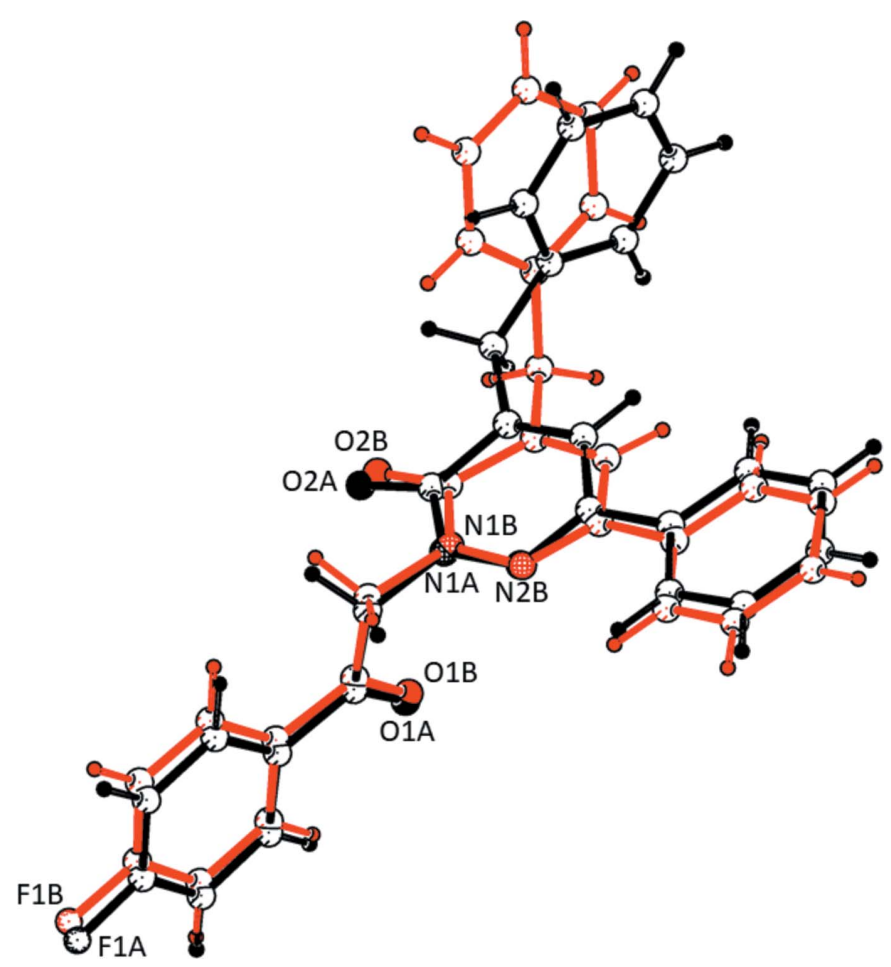

Figure 2

A structural overlap view of molecule $A$ (black) on molecule $B$ (red), drawn using PLATON (Spek, 2009).

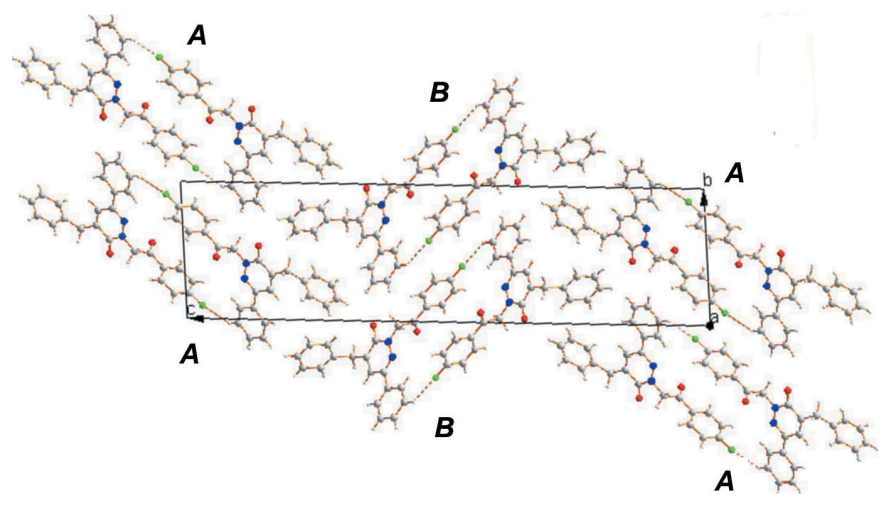

Figure 3

A view along the $a$ axis of the crystal packing of the title compound. The $\mathrm{C}-\mathrm{H} \cdots \mathrm{F}$ hydrogen bonds are shown as dashed lines (see Table 1 ). 
molecules are linked to each other in a similar manner (Table 1), and also form columns separating the columns of $A$ molecules, as illustrated in Fig. 3.

\section{Frontier molecular orbitals analysis}

The highest occupied molecular orbitals (HOMOs) and the lowest-lying unoccupied molecular orbitals (LUMOs) are named as frontier molecular orbitals (FMOs). The FMOs play an important role in the optical and electric properties, as well as in quantum chemistry and UV-vis spectra. As a result of the interaction between the HOMO and LUMO orbitals of a structure, a transition state of the $\pi-\pi^{*}$ type is observed according to molecular orbital theory. The frontier orbital gap helps characterize the chemical reactivity and the kinetic stability of the molecule. A molecule with a small frontier orbital gap is generally associated with a high chemical reactivity, low kinetic stability and is also termed as a soft molecule. The DFT quantum-chemical calculations for the title compound were performed at the B3LYP/6-311 G(d,p) level (Becke, 1993) as implemented in GAUSSIAN09 (Frisch et al., 2009). The DFT structure optimization was performed starting from the X-ray geometry and the experimental values of the bond lengths and bond angles match the theoretical values. The DFT study shows that the HOMO and LUMO are localized in the plane extending from the whole substituted oxopyridazinyl ring. The electron distribution of the HOMO-1, HOMO, LUMO and LUMO+1 energy levels is

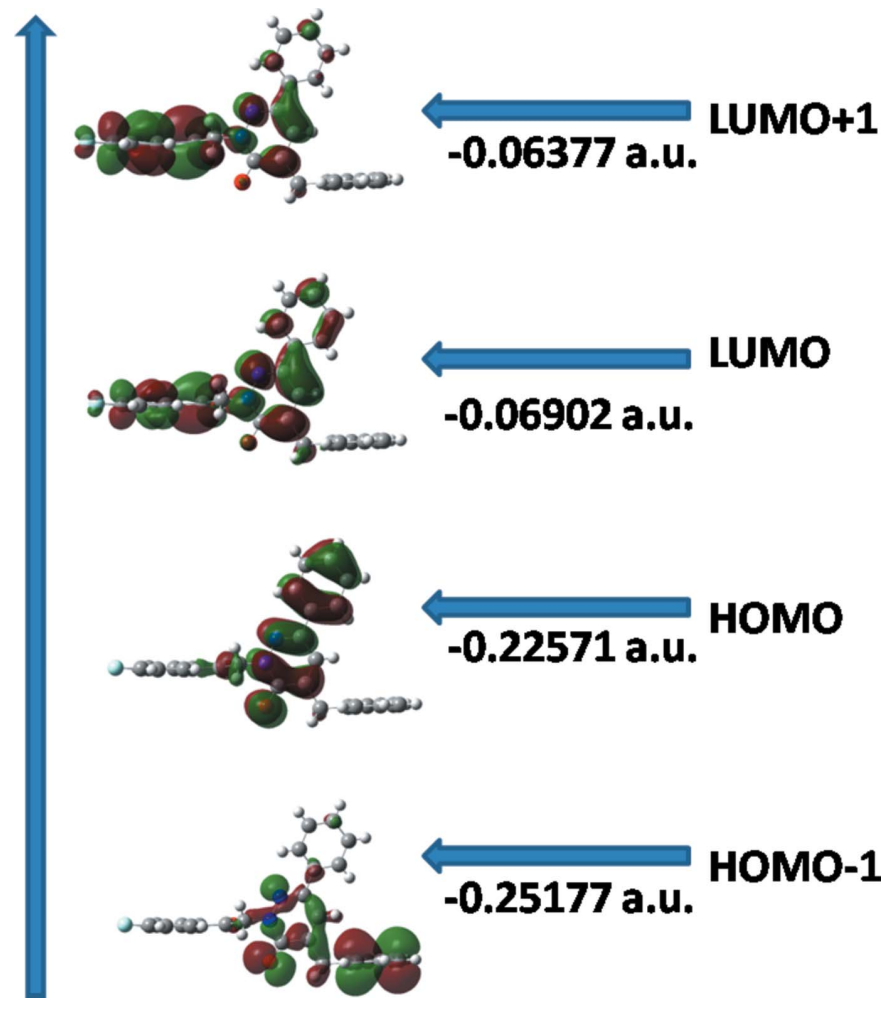

Figure 4

Electron distribution of the HOMO-1, HOMO, LUMO and the $\mathrm{LUMO}+1$ energy levels for the title compound. shown in Fig. 4. The HOMO molecular orbital exhibits both $\sigma$ and $\pi$ character, whereas HOMO- 1 is dominated by $\pi$-orbital density. The LUMO is mainly composed of $\pi$-density while LUMO +1 has both $\sigma$ and $\pi$ electronic density. The HOMOLUMO gap is 0.15669 a.u. and the frontier molecular orbital energies, $E_{\mathrm{HOMO}}$ and $E_{\mathrm{LUMO}}$ are -0.22571 and -0.06902 a.u., respectively.

\section{Molecular electrostatic potential surface analysis}

The molecular electrostatic potential (MEP) is a technique of mapping electrostatic potential onto the iso-electron density surface. The MEP surface provides information about the reactive sites. The colour scheme is as follows: red for electron rich, partial negative charge; blue for electron-deficient, partial positive charge; light blue for a slightly electron deficient region; yellow for a slightly electron-rich region; green for neutral (Politzer \& Murray, 2002). In addition to these, in the majority of the MEPs, while the maximum positive region, which is the preferred site for nucleophilic attack, is indicated in blue, the maximum negative region, which is the preferred site for electrophilic attack, is indicated in red. The threedimensional plot of the MEP of the title compound is shown in Fig. 5. According to the MEP map results, the negative regions of the whole molecule are located on donor oxygen atoms (red regions). The resulting surface simultaneously displays the molecular size and shape and electrostatic potential values. As can be seen from the MEP map contours, regions having negative potential are over the electronegative atoms ( $v i z$. atoms $\mathrm{O} 1 A$ and $\mathrm{O} 2 A$ of molecule $A$ and $\mathrm{O} 1 B$ and $\mathrm{O} 2 B$ of molecule $B$ ). The positive regions are over hydrogen atoms, indicating that these sites are the most likely to be involved in nucleophilic processes.

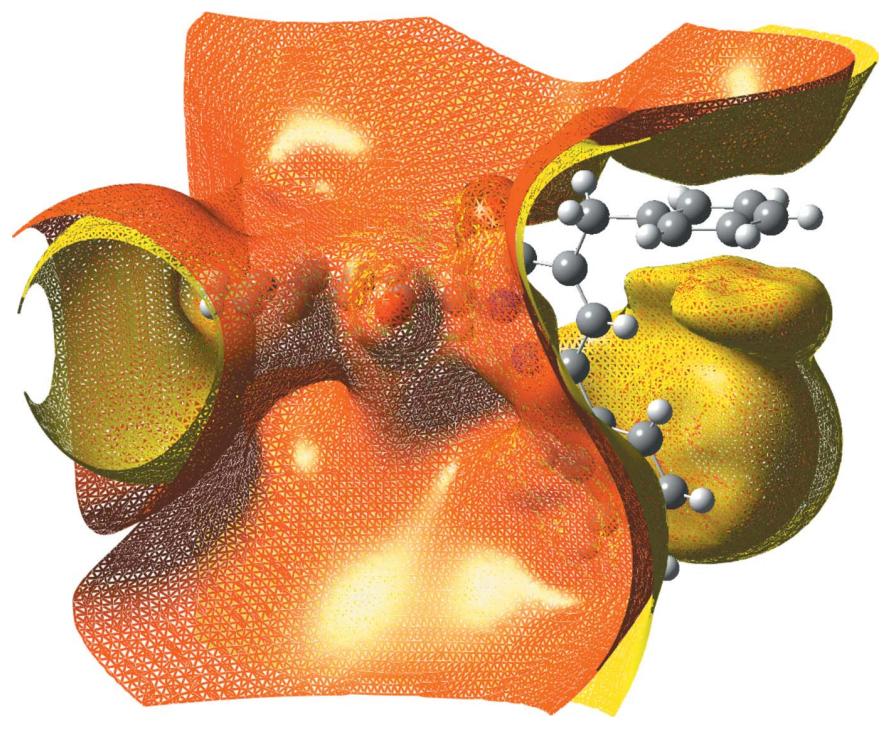

Figure 5

Total electron density mapped over the molecular electrostatic potential surface of the title compound. 
Table 2

Experimental details.

\begin{tabular}{|c|c|}
\hline \multicolumn{2}{|l|}{ Crystal data } \\
\hline Chemical formula & $\mathrm{C}_{25} \mathrm{H}_{19} \mathrm{FN}_{2} \mathrm{O}_{2}$ \\
\hline$M_{\mathrm{r}}$ & 398.42 \\
\hline Crystal system, space group & Triclinic, $P \overline{1}$ \\
\hline Temperature $(\mathrm{K})$ & 296 \\
\hline$a, b, c(\AA)$ & $5.0575(3), 10.0973(7), 38.608(2)$ \\
\hline$\alpha, \beta, \gamma\left({ }^{\circ}\right)$ & $86.237(5), 86.675(5), 88.354(5)$ \\
\hline$V\left(\AA^{3}\right)$ & $1963.4(2)$ \\
\hline$Z$ & 4 \\
\hline Radiation type & Мо $K \alpha$ \\
\hline$\mu\left(\mathrm{mm}^{-1}\right)$ & 0.09 \\
\hline Crystal size $(\mathrm{mm})$ & $0.67 \times 0.53 \times 0.44$ \\
\hline \multicolumn{2}{|l|}{ Data collection } \\
\hline Diffractometer & Stoe IPDS 2 \\
\hline Absorption correction & $\begin{array}{l}\text { Integration ( } X \text {-RED32; Stoe \& Cie, } \\
\text { 2002) }\end{array}$ \\
\hline$T_{\min }, T_{\max }$ & $0.953,0.974$ \\
\hline $\begin{array}{l}\text { No. of measured, independent and } \\
\text { observed }[I>2 \sigma(I)] \text { reflections }\end{array}$ & $16344,6363,4315$ \\
\hline$R_{\text {int }}$ & 0.031 \\
\hline$(\sin \theta / \lambda)_{\max }\left(\AA^{-1}\right)$ & 0.582 \\
\hline \multicolumn{2}{|l|}{ Refinement } \\
\hline$R\left[F^{2}>2 \sigma\left(F^{2}\right)\right], w R\left(F^{2}\right), S$ & $0.038,0.108,0.99$ \\
\hline No. of reflections & 6363 \\
\hline No. of parameters & 542 \\
\hline $\mathrm{H}$-atom treatment & H-atom parameters constrained \\
\hline$\Delta \rho_{\max }, \Delta \rho_{\min }\left(\mathrm{e} \AA^{-3}\right)$ & $0.16,-0.15$ \\
\hline
\end{tabular}

Computer programs: $X$-AREA and X-RED32 (Stoe \& Cie, 2002), SHELXT2018 (Sheldrick, 2015a), SHELXL2018 (Sheldrick, 2015b), ORTEP-3 for Windows and WinGX (Farrugia, 2012), PLATON (Spek, 2009) and Mercury (Macrae et al., 2008).

\section{Database survey}

A search of the Cambridge Structural Database (CSD, version 5.40, update February 2019; Groom et al., 2016) gave zero hits for the skeleton of the title compound. A search for pyridazin$3(2 H)$-ones gave 297 hits, while a search for 6-phenyl-pyridazin-3(2H)-ones gave 40 hits, including 6-phenyl-pyridazin$3(2 \mathrm{H})$-one itself (CSD refcode CUBBOR; Anderson et al., 2009). A search for 4-benzyl-6-phenyl-pyridazin-3(2H)-ones gave only three hits, for example 4-(4-bromobenzyl)-6phenylpyridazin-3(2H)-one (VOPMOE; Tsai et al., 2014). A search for pyridazin-3(2H)-ones with an oxoethyl group in position-2 on the pyridazine ring gave eight hits, mostly esters. Four of these structures also have a phenyl substituent in position- 6 on the pyridazine ring, as in the title compound. They include, for example 2-(6-oxo-3,4-diphenyl-1,6-dihydropyridazin-1-yl)acetic acid (CIPTOL; Aydın et al., 2007).

\section{Synthesis and crystallization}

A mixture of 4-benzyl-6-phenylpyridazin-3(2H)-one (1 g, $3.8 \mathrm{mmol}), \mathrm{K}_{2} \mathrm{CO}_{3}(1.3 \mathrm{~g}, 9.5 \mathrm{mmol}$ ) and 2-chloro-1-(4-fluorophenyl)ethan-1-one $(1.58 \mathrm{~g}, 5 \mathrm{mmol})$ in acetone $(40 \mathrm{ml})$, was refluxed overnight. The solution was then filtered by suction and the solvent removed under reduced pressure. The residue was purified by recrystallization from ethanol to afford the title compound as colourless prismatic crystals (yield 68\%).

\section{Refinement}

Crystal data, data collection and structure refinement details are summarized in Table 2. The carbon-bound $\mathrm{H}$ atoms were placed in calculated positions $(\mathrm{C}-\mathrm{H}=0.93-0.97 \AA)$ and included in the refinement in the riding-model approximation, with $U_{\text {iso }}(\mathrm{H})=1.2 U_{\text {eq }}(\mathrm{C})$. The image plate disc in the diffractometer used for the data collection was unfortunately distorted at the outer edges, hence the maximum $2 \theta$ value available was limited to $48.8^{\circ}$.

\section{Acknowledgements}

This study was supported by Hassan II University, Casablanca, Morocco, Mohammed V University, Rabat, Morocco and Langat Singh College, BRABU, Muzaffarpur, India.

\section{Funding information}

Funding for this research was provided by a start-up grant from the University Grants Commission, India.

\section{References}

Abiha, G. B., Bahar, L. \& Utku, S. (2018). Rev. Rom. Med. Lab. 26, 231-241.

Anderson, K. M., Probert, M. R., Whiteley, C. N., Rowland, A. M., Goeta, A. E. \& Steed, J. W. (2009). Cryst. Growth Des. 9, 10821087.

Aydın, A., Doğruer, D. S., Akkurt, M. \& Büyükgüngör, O. (2007). Acta Cryst. E63, o4522.

Azaari, H., Chahboune, R., El Azzouzi, M. \& Sarakha, M. (2016). Rapid Commun. Mass Spectrom. 30, 1145-1152.

Becke, A. D. (1993). J. Chem. Phys. 98, 5648-5652.

Boukharsa, Y., Meddah, B., Tiendrebeogo, R. Y., Ibrahimi, A., Taoufik, J., Cherrah, Y., Benomar, A., Faouzi, M. E. A. \& Ansar, M. (2016). Med. Chem. Res. 25, 494-500.

Faizi, M. S. H., Gupta, S., Mohan, V. K., Jain, K. V. \& Sen, P. (2016). Sens. Actuators B Chem. 222, 15-20.

Farrugia, L. J. (2012). J. Appl. Cryst. 45, 849-854.

Frisch, M. J., Trucks, G. W., Schlegel, H. B., Scuseria, G. E., Robb, M. A., Cheeseman, J. R., et al. (2009). GAUSSIAN09. Gaussian Inc., Wallingford, CT, USA.

Groom, C. R., Bruno, I. J., Lightfoot, M. P. \& Ward, S. C. (2016). Acta Cryst. B72, 171-179.

Ibrahim, T. H., Loksha, Y. M., Elshihawy, H. A., Khodeer, D. M. \& Said, M. M. (2017). Arch. Pharm. Chem. Life Sci. 350, e1700093.

Igarashi, H. \& Sakamoto, S. (1994). J. Pestic. Sci. 19, S243-S251.

Kamble, V. T., Sawant, A.-S., Sawant, S. S., Pisal, P. M., Gacche, R. N., Kamble, S. S., Shegokar, H. D. \& Kamble, V. A. (2017). J. Basic Appl. Res. Int, 21, 10-39.

Khokra, S. L., Khan, S. A., Thakur, P., Chowdhary, D., Ahmad, A. \& Husain, A. (2016). J. Chin. Chem. Soc. 63, 739-750.

Kumar, M., Kumar, A., Faizi, M. S. H., Kumar, S., Singh, M. K., Sahu, S. K., Kishor, S. \& John, R. P. (2018). Sens. Actuators B Chem. 260, 888-899.

Kumar, S., Hansda, A., Chandra, A., Kumar, A., Kumar, M., Sithambaresan, M., Faizi, M. S. H., Kumar, V. \& John, R. P. (2017). Polyhedron, 134, 11-21.

Macrae, C. F., Bruno, I. J., Chisholm, J. A., Edgington, P. R., McCabe, P., Pidcock, E., Rodriguez-Monge, L., Taylor, R., van de Streek, J. \& Wood, P. A. (2008). J. Appl. Cryst. 41, 466-470.

Mukherjee, P., Das, A., Faizi, M. S. H. \& Sen, P. (2018). Chemistry Select, 3, 3787-3796.

Nauen, R. \& Bretschneider, T. (2002). Pest. Outlook, 13, 241-245.

Politzer, P. \& Murray, J. S. (2002). Theor. Chim. Acta, 108, 134-142. 
Sheldrick, G. M. (2015a). Acta Cryst. A71, 3-8.

Sheldrick, G. M. (2015b). Acta Cryst. C71, 3-8.

Spek, A. L. (2009). Acta Cryst. D65, 148-155.

Stoe \& Cie (2002). $X$-AREA, X-RED32 and X-SHAPE. Stoe \& Cie $\mathrm{GmbH}$, Darmstadt, Germany.
Tsai, Y.-L., Syu, S.-E., Yang, S.-M., Das, U., Fan, Y.-S., Lee, C.-J. \& Lin, W. (2014). Tetrahedron, 70, 5038-5045.

Yamada, T., Nobuhara, Y., Shimamura, H., Yoshihara, K., Yamaguchi, A. \& Ohki, M. (1981). Chem. Pharm. Bull. 29, 3433-3439. 


\section{supporting information}

Acta Cryst. (2019). E75, 1030-1034 [https://doi.org/10.1107/S2056989019008557]

Crystal structure and the DFT and MEP study of 4-benzyl-2-[2-(4-fluorophenyl)-2-oxoethyl]-6-phenylpyridazin-3(2H)-one

Said Daoui, Md. Serajul Haque Faizi, Fouad El Kalai, Rafik Saddik, Necmi Dege, Khalid

Karrouchi and Noureddine Benchat

Computing details

Data collection: $X$-AREA (Stoe \& Cie, 2002); cell refinement: $X$-AREA (Stoe \& Cie, 2002); data reduction: $X$-RED32

(Stoe \& Cie, 2002); program(s) used to solve structure: SHELXT2018 (Sheldrick, 2015a); program(s) used to refine structure: SHELXL2018 (Sheldrick, 2015b); molecular graphics: ORTEP-3 for Windows (Farrugia, 2012), PLATON

(Spek, 2009) and Mercury (Macrae et al., 2008); software used to prepare material for publication: SHELXL2018

(Sheldrick, 2015b), WinGX (Farrugia, 2012) and PLATON (Spek, 2009).

4-Benzyl-2-[2-(4-fluorophenyl)-2-oxoethyl]-6-phenylpyridazin-3(2H)-one

Crystal data

$\mathrm{C}_{25} \mathrm{H}_{19} \mathrm{FN}_{2} \mathrm{O}_{2}$

$M_{r}=398.42$

Triclinic, $P \overline{1}$

$a=5.0575(3) \AA$

$b=10.0973(7) \AA$

$c=38.608(2) \AA$

$\alpha=86.237(5)^{\circ}$

$\beta=86.675(5)^{\circ}$

$\gamma=88.354(5)^{\circ}$

$V=1963.4(2) \AA^{3}$

$$
\begin{aligned}
& Z=4 \\
& F(000)=832
\end{aligned}
$$

$D_{\mathrm{x}}=1.348 \mathrm{Mg} \mathrm{m}^{-3}$

Mo $K \alpha$ radiation, $\lambda=0.71073 \AA$

Cell parameters from 17316 reflections

$\theta=1.1-25.0^{\circ}$

$\mu=0.09 \mathrm{~mm}^{-1}$

$T=296 \mathrm{~K}$

Prism, colourless

$0.67 \times 0.53 \times 0.44 \mathrm{~mm}$

Data collection

Stoe IPDS 2

diffractometer

Radiation source: sealed X-ray tube, 12 x 0.4 $\mathrm{mm}$ long-fine focus

Plane graphite monochromator

Detector resolution: 6.67 pixels $\mathrm{mm}^{-1}$

rotation method scans

Absorption correction: integration

(X-RED32; Stoe \& Cie, 2002)

$T_{\min }=0.953, T_{\max }=0.974$

16344 measured reflections

6363 independent reflections

4315 reflections with $I>2 \sigma(I)$

$R_{\text {int }}=0.031$

$\theta_{\max }=24.4^{\circ}, \theta_{\min }=1.1^{\circ}$

$h=-5 \rightarrow 5$

$k=-11 \rightarrow 11$

$l=-44 \rightarrow 44$

Refinement

Refinement on $F^{2}$

Least-squares matrix: full

$R\left[F^{2}>2 \sigma\left(F^{2}\right)\right]=0.038$

$w R\left(F^{2}\right)=0.108$

$S=0.99$

6363 reflections

542 parameters

0 restraints

Primary atom site location: structure-invariant direct methods 
Secondary atom site location: difference Fourier map

Hydrogen site location: inferred from neighbouring sites

$\mathrm{H}$-atom parameters constrained

$w=1 /\left[\sigma^{2}\left(F_{\mathrm{o}}^{2}\right)+(0.0633 P)^{2}\right]$

where $P=\left(F_{\mathrm{o}}^{2}+2 F_{\mathrm{c}}^{2}\right) / 3$

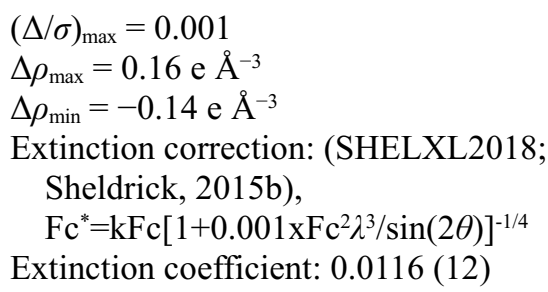

Special details

Geometry. All esds (except the esd in the dihedral angle between two 1.s. planes) are estimated using the full covariance matrix. The cell esds are taken into account individually in the estimation of esds in distances, angles and torsion angles; correlations between esds in cell parameters are only used when they are defined by crystal symmetry. An approximate (isotropic) treatment of cell esds is used for estimating esds involving 1.s. planes.

Fractional atomic coordinates and isotropic or equivalent isotropic displacement parameters $\left(\AA^{2}\right)$

\begin{tabular}{|c|c|c|c|c|}
\hline & $x$ & $y$ & $z$ & $U_{\text {iso }} * / U_{\text {eq }}$ \\
\hline $\mathrm{F} 1 \mathrm{~A}$ & $0.6289(5)$ & $0.09624(17)$ & -0.02980 & $0.1608(8)$ \\
\hline O1A & $0.1611(3)$ & $0.54893(15)$ & 0.06085 & $0.0884(4)$ \\
\hline $\mathrm{O} 2 \mathrm{~A}$ & $0.1102(3)$ & $0.46400(14)$ & $0.14261(3)$ & $0.0843(4)$ \\
\hline N1A & 0.4405 & $0.59106(15)$ & $0.11746(4)$ & $0.0671(4)$ \\
\hline $\mathrm{N} 2 \mathrm{~A}$ & 0.5835 & $0.70263(15)$ & $0.11242(4)$ & $0.0647(4)$ \\
\hline $\mathrm{C} 1 \mathrm{~A}$ & $0.5570(7)$ & 0.1845 & $-0.00606(7)$ & $0.1070(9)$ \\
\hline $\mathrm{C} 2 \mathrm{~A}$ & $0.3566(7)$ & $0.2716(3)$ & $-0.01297(6)$ & $0.1131(9)$ \\
\hline $\mathrm{H} 2 \mathrm{~A}$ & 0.267897 & 0.269609 & -0.033355 & $0.136^{*}$ \\
\hline $\mathrm{C} 3 \mathrm{~A}$ & $0.2873(5)$ & $0.3632(2)$ & $0.01093(6)$ & $0.0928(7)$ \\
\hline $\mathrm{H} 3 \mathrm{~A}$ & 0.147897 & 0.423052 & 0.006716 & $0.111^{*}$ \\
\hline $\mathrm{C} 4 \mathrm{~A}$ & $0.4204(4)$ & 0.36847 (19) & $0.04125(5)$ & $0.0682(5)$ \\
\hline $\mathrm{C} 5 \mathrm{~A}$ & $0.6218(5)$ & $0.2767(2)$ & $0.04710(6)$ & $0.0879(6)$ \\
\hline $\mathrm{H} 5 \mathrm{~A}$ & 0.713023 & 0.277567 & 0.067316 & $0.105^{*}$ \\
\hline C6A & $0.6904(6)$ & $0.1834(3)$ & $0.02333(7)$ & $0.1084(8)$ \\
\hline H6A & 0.825601 & 0.120960 & 0.027461 & $0.130 *$ \\
\hline C7A & $0.3491(4)$ & 0.47327 (19) & $0.06528(5)$ & $0.0674(5)$ \\
\hline C8A & $0.5252(4)$ & 0.48597 (19) & $0.09515(5)$ & $0.0716(5)$ \\
\hline $\mathrm{H} 8 \mathrm{~A}$ & 0.528879 & 0.402357 & 0.109027 & $0.086^{*}$ \\
\hline H8B & 0.704330 & 0.502655 & 0.085854 & $0.086 *$ \\
\hline C9A & $0.2332(4)$ & 0.56748 (19) & $0.14188(5)$ & $0.0668(5)$ \\
\hline C10A & $0.1890(4)$ & 0.67082 (19) & $0.16591(4)$ & $0.0643(5)$ \\
\hline C11A & $0.3298(3)$ & 0.78185 (19) & $0.16100(4)$ & $0.0638(4)$ \\
\hline H11A & 0.296983 & 0.849831 & 0.175921 & $0.077^{*}$ \\
\hline C12A & 0.5292 & $0.79793(17)$ & 0.13333 & $0.0590(4)$ \\
\hline C13A & $0.6973(3)$ & 0.91648 (17) & 0.12757 (4) & $0.0592(4)$ \\
\hline C14A & $0.9022(4)$ & $0.9212(2)$ & $0.10193(5)$ & $0.0701(5)$ \\
\hline H14A & 0.930936 & 0.850425 & 0.087826 & $0.084 *$ \\
\hline $\mathrm{C} 15 \mathrm{~A}$ & $1.0634(4)$ & $1.0296(2)$ & $0.09714(5)$ & $0.0785(5)$ \\
\hline H15A & 1.198796 & 1.031103 & 0.079764 & $0.094 *$ \\
\hline C16A & $1.0263(4)$ & $1.1351(2)$ & $0.11770(5)$ & $0.0771(5)$ \\
\hline H16A & 1.135777 & 1.207810 & 0.114378 & $0.093 *$ \\
\hline C17A & $0.8272(4)$ & $1.1320(2)$ & $0.14305(5)$ & $0.0800(6)$ \\
\hline
\end{tabular}




\begin{tabular}{|c|c|c|c|c|}
\hline H17A & 0.801440 & 1.202863 & 0.157184 & $0.096^{*}$ \\
\hline C18A & $0.6631(4)$ & $1.02435(19)$ & $0.14795(5)$ & $0.0737(5)$ \\
\hline H18A & 0.527209 & 1.024344 & 0.165248 & $0.088^{*}$ \\
\hline C19A & $-0.0153(4)$ & $0.6428(2)$ & $0.19543(5)$ & $0.0776(5)$ \\
\hline H19A & -0.189465 & 0.665208 & 0.187160 & $0.093 *$ \\
\hline H19B & -0.010103 & 0.548385 & 0.201910 & $0.093 *$ \\
\hline $\mathrm{C} 20 \mathrm{~A}$ & $0.0200(4)$ & $0.7165(2)$ & $0.22721(5)$ & $0.0748(5)$ \\
\hline $\mathrm{C} 21 \mathrm{~A}$ & $-0.1352(5)$ & $0.8225(3)$ & $0.23581(7)$ & $0.1061(8)$ \\
\hline $\mathrm{H} 21 \mathrm{~A}$ & -0.268536 & 0.853174 & 0.221492 & $0.127 *$ \\
\hline $\mathrm{C} 22 \mathrm{~A}$ & $-0.0946(9)$ & $0.8864(3)$ & $0.26656(11)$ & $0.1434(13)$ \\
\hline $\mathrm{H} 22 \mathrm{~A}$ & -0.200439 & 0.959078 & 0.272578 & $0.172 *$ \\
\hline $\mathrm{C} 23 \mathrm{~A}$ & $0.1034(11)$ & $0.8396(5)$ & $0.28740(9)$ & $0.1518(19)$ \\
\hline $\mathrm{H} 23 \mathrm{~A}$ & 0.131088 & 0.880473 & 0.307731 & $0.182 *$ \\
\hline $\mathrm{C} 24 \mathrm{~A}$ & $0.2553(8)$ & $0.7361(5)$ & $0.27864(8)$ & $0.1443(14)$ \\
\hline $\mathrm{H} 24$ & 0.390783 & 0.705923 & 0.292665 & $0.173^{*}$ \\
\hline $\mathrm{C} 25 \mathrm{~A}$ & $0.2138(5)$ & $0.6744(3)$ & $0.24941(6)$ & $0.1052(8)$ \\
\hline $\mathrm{H} 25 \mathrm{~A}$ & 0.319992 & 0.600939 & 0.244112 & $0.126^{*}$ \\
\hline F1B & $-0.1157(4)$ & $-0.40818(14)$ & $0.53084(4)$ & $0.1266(5)$ \\
\hline O1B & $0.3487(3)$ & $0.07220(14)$ & $0.43808(3)$ & $0.0772(4)$ \\
\hline $\mathrm{O} 2 \mathrm{~B}$ & $0.4021(3)$ & $0.03214(13)$ & $0.35481(3)$ & $0.0802(4)$ \\
\hline N1B & $0.0734(3)$ & $0.15026(13)$ & $0.38207(3)$ & $0.0584(4)$ \\
\hline $\mathrm{N} 2 \mathrm{~B}$ & $-0.0726(3)$ & $0.26127(13)$ & $0.38851(3)$ & $0.0555(3)$ \\
\hline $\mathrm{C} 1 \mathrm{~B}$ & $-0.0437(5)$ & $-0.3100(2)$ & $0.50691(5)$ & $0.0843(6)$ \\
\hline $\mathrm{C} 2 \mathrm{~B}$ & $0.1540(5)$ & $-0.2291(2)$ & $0.51344(5)$ & $0.0873(6)$ \\
\hline $\mathrm{H} 2 \mathrm{~B}$ & 0.242165 & -0.240984 & 0.533934 & $0.105^{*}$ \\
\hline $\mathrm{C} 3 \mathrm{~B}$ & $0.2203(4)$ & $-0.1293(2)$ & $0.48904(5)$ & $0.0751(5)$ \\
\hline H3B & 0.356166 & -0.073010 & 0.493036 & $0.090^{*}$ \\
\hline $\mathrm{C} 4 \mathrm{~B}$ & $0.0889(3)$ & $-0.11043(16)$ & $0.45843(4)$ & $0.0563(4)$ \\
\hline $\mathrm{C} 5 \mathrm{~B}$ & $-0.1091(4)$ & $-0.19582(19)$ & $0.45264(5)$ & $0.0710(5)$ \\
\hline H5B & -0.198472 & -0.184832 & 0.432219 & $0.085^{*}$ \\
\hline $\mathrm{C} 6 \mathrm{~B}$ & $-0.1761(5)$ & $-0.2978(2)$ & $0.47698(6)$ & $0.0842(6)$ \\
\hline H6B & -0.307935 & -0.356518 & 0.473060 & $0.101 *$ \\
\hline C7B & 0.1617 (3) & $0.00317(17)$ & $0.43376(4)$ & $0.0573(4)$ \\
\hline $\mathrm{C} 8 \mathrm{~B}$ & $-0.0105(3)$ & $0.03345(16)$ & $0.40320(4)$ & $0.0611(4)$ \\
\hline $\mathrm{H} 8 \mathrm{C}$ & -0.004029 & -0.042157 & 0.388888 & $0.073^{*}$ \\
\hline H8D & -0.192703 & 0.046919 & 0.411803 & $0.073^{*}$ \\
\hline C9B & $0.2882(3)$ & $0.14027(18)$ & $0.35847(4)$ & $0.0601(4)$ \\
\hline C10B & $0.3580(3)$ & $0.26374(17)$ & $0.33930(4)$ & $0.0562(4)$ \\
\hline $\mathrm{C} 11 \mathrm{~B}$ & $0.2149(3)$ & $0.37419(17)$ & $0.34604(4)$ & $0.0561(4)$ \\
\hline H11B & 0.261247 & 0.454364 & 0.334336 & $0.067^{*}$ \\
\hline $\mathrm{C} 12 \mathrm{~B}$ & $-0.0065(3)$ & $0.37147(16)$ & $0.37073(4)$ & $0.0514(4)$ \\
\hline C13B & $-0.1834(3)$ & $0.48873(15)$ & $0.37667(4)$ & $0.0525(4)$ \\
\hline C14B & $-0.3844(3)$ & $0.48391(18)$ & $0.40284(4)$ & $0.0616(4)$ \\
\hline H14B & -0.402871 & 0.407558 & 0.417523 & $0.074 *$ \\
\hline $\mathrm{C} 15 \mathrm{~B}$ & $-0.5563(4)$ & $0.59007(18)$ & $0.40738(5)$ & $0.0690(5)$ \\
\hline H15B & -0.689600 & 0.584914 & 0.425007 & $0.083^{*}$ \\
\hline $\mathrm{C} 16 \mathrm{~B}$ & $-0.5320(4)$ & 0.70395 (19) & $0.38596(5)$ & $0.0683(5)$ \\
\hline H16B & -0.648412 & 0.775698 & 0.389098 & $0.082 *$ \\
\hline
\end{tabular}




$\begin{array}{lllll}\text { C17B } & -0.3363(4) & 0.71117(18) & 0.36007(5) & 0.0688(5) \\ \text { H17B } & -0.319755 & 0.787943 & 0.345510 & 0.083^{*} \\ \text { C18B } & -0.1626(4) & 0.60469(17) & 0.35541(4) & 0.0643(5) \\ \text { H18B } & -0.029726 & 0.610886 & 0.337740 & 0.077^{*} \\ \text { C19B } & 0.5868(3) & 0.2626(2) & 0.31260(4) & 0.0663(5) \\ \text { H19C } & 0.697238 & 0.184128 & 0.317408 & 0.080^{*} \\ \text { H19D } & 0.693213 & 0.339614 & 0.314856 & 0.080^{*} \\ \text { C20B } & 0.5043(3) & 0.26336(16) & 0.27574(4) & 0.0559(4) \\ \text { C21B } & 0.6112(5) & 0.3470(2) & 0.25011(5) & 0.0966(7) \\ \text { H21B } & 0.740478 & 0.405093 & 0.255303 & 0.116^{*} \\ \text { C22B } & 0.5316(6) & 0.3476(3) & 0.21622(6) & 0.1098(9) \\ \text { H22B } & 0.606266 & 0.407224 & 0.199291 & 0.132^{*} \\ \text { C23B } & 0.3522(5) & 0.2651(2) & 0.20764(5) & 0.0839(6) \\ \text { H23B } & 0.302079 & 0.265730 & 0.184825 & 0.101^{*} \\ \text { C24B } & 0.2429(6) & 0.1804(3) & 0.23227(6) & 0.1173(9) \\ \text { H24B } & 0.115371 & 0.122164 & 0.226601 & 0.141^{*} \\ \text { C25B } & 0.3195(5) & 0.1796(3) & 0.26598(6) & 0.1101(9) \\ \text { H25B } & 0.242238 & 0.119871 & 0.282659 & 0.132^{*}\end{array}$

Atomic displacement parameters $\left(\AA^{2}\right)$

\begin{tabular}{lllllll}
\hline & $U^{11}$ & $U^{22}$ & $U^{33}$ & $U^{12}$ & $U^{13}$ & $U^{23}$ \\
\hline F1A & $0.256(2)$ & $0.1202(12)$ & $0.1074(11)$ & $-0.0320(13)$ & $0.0419(12)$ & $-0.0539(9)$ \\
O1A & $0.0780(9)$ & $0.0997(11)$ & $0.0865(9)$ & $0.0114(9)$ & $-0.0045(7)$ & $-0.0049(8)$ \\
O2A & $0.0967(10)$ & $0.0754(9)$ & $0.0813(9)$ & $-0.0261(8)$ & $-0.0038(7)$ & $-0.0001(7)$ \\
N1A & $0.0673(9)$ & $0.0631(9)$ & $0.0712(9)$ & $-0.0060(8)$ & $-0.0003(8)$ & $-0.0088(7)$ \\
N2A & $0.0624(9)$ & $0.0636(9)$ & $0.0682(9)$ & $-0.0079(8)$ & $-0.0010(7)$ & $-0.0053(7)$ \\
C1A & $0.161(3)$ & $0.0827(17)$ & $0.0773(15)$ & $-0.0335(18)$ & $0.0291(16)$ & $-0.0238(13)$ \\
C2A & $0.164(3)$ & $0.104(2)$ & $0.0745(15)$ & $-0.040(2)$ & $-0.0073(16)$ & $-0.0167(14)$ \\
C3A & $0.1055(17)$ & $0.0920(15)$ & $0.0825(14)$ & $-0.0137(13)$ & $-0.0141(13)$ & $-0.0050(12)$ \\
C4A & $0.0727(12)$ & $0.0679(12)$ & $0.0635(11)$ & $-0.0170(10)$ & $0.0053(9)$ & $-0.0026(9)$ \\
C5A & $0.1025(17)$ & $0.0828(14)$ & $0.0793(13)$ & $0.0020(13)$ & $-0.0038(12)$ & $-0.0167(11)$ \\
C6A & $0.130(2)$ & $0.0906(17)$ & $0.1048(19)$ & $0.0070(15)$ & $0.0092(16)$ & $-0.0282(14)$ \\
C7A & $0.0631(11)$ & $0.0694(12)$ & $0.0683(11)$ & $-0.0107(10)$ & $0.0063(9)$ & $0.0019(9)$ \\
C8A & $0.0708(12)$ & $0.0643(11)$ & $0.0806(12)$ & $-0.0020(10)$ & $-0.0027(10)$ & $-0.0144(9)$ \\
C9A & $0.0694(12)$ & $0.0662(12)$ & $0.0649(10)$ & $-0.0094(10)$ & $-0.0080(9)$ & $0.0020(9)$ \\
C10A & $0.0600(10)$ & $0.0739(12)$ & $0.0588(10)$ & $-0.0085(10)$ & $-0.0055(8)$ & $0.0013(9)$ \\
C11A & $0.0615(11)$ & $0.0700(12)$ & $0.0601(10)$ & $-0.0042(9)$ & $-0.0003(8)$ & $-0.0082(8)$ \\
C12A & $0.0568(10)$ & $0.0637(11)$ & $0.0567(9)$ & $0.0001(9)$ & $-0.0029(8)$ & $-0.0060(8)$ \\
C13A & $0.0563(10)$ & $0.0643(11)$ & $0.0574(9)$ & $-0.0026(8)$ & $-0.0050(8)$ & $-0.0041(8)$ \\
C14A & $0.0710(12)$ & $0.0773(12)$ & $0.0627(10)$ & $-0.0117(10)$ & $0.0048(9)$ & $-0.0126(9)$ \\
C15A & $0.0725(13)$ & $0.0916(15)$ & $0.0711(11)$ & $-0.0194(11)$ & $0.0082(9)$ & $-0.0056(11)$ \\
C16A & $0.0745(13)$ & $0.0764(13)$ & $0.0816(13)$ & $-0.0196(11)$ & $-0.0055(11)$ & $-0.0057(11)$ \\
C17A & $0.0854(14)$ & $0.0706(13)$ & $0.0855(13)$ & $-0.0117(11)$ & $0.0015(11)$ & $-0.0189(10)$ \\
C18A & $0.0726(12)$ & $0.0714(12)$ & $0.0769(12)$ & $-0.0078(10)$ & $0.0097(10)$ & $-0.0130(10)$ \\
C19A & $0.0666(12)$ & $0.0979(15)$ & $0.0677(11)$ & $-0.0175(11)$ & $0.0021(9)$ & $-0.0003(10)$ \\
C20A & $0.0664(12)$ & $0.0952(15)$ & $0.0616(11)$ & $-0.0213(11)$ & $0.0083(9)$ & $0.0020(10)$ \\
C21A & $0.0987(18)$ & $0.1080(19)$ & $0.1115(19)$ & $-0.0175(16)$ & $0.0124(15)$ & $-0.0148(16)$ \\
& & & & & &
\end{tabular}




\begin{tabular}{|c|c|c|c|c|c|c|}
\hline $\mathrm{C} 22 \mathrm{~A}$ & $0.171(3)$ & $0.115(2)$ & $0.143(3)$ & $-0.046(2)$ & $0.055(3)$ & $-0.041(2)$ \\
\hline $\mathrm{C} 23 \mathrm{~A}$ & $0.202(5)$ & $0.181(4)$ & $0.077(2)$ & $-0.119(4)$ & $0.030(2)$ & $-0.023(2)$ \\
\hline $\mathrm{C} 24 \mathrm{~A}$ & $0.154(3)$ & $0.216(4)$ & $0.0664(18)$ & $-0.082(3)$ & $-0.0151(17)$ & $0.010(2)$ \\
\hline $\mathrm{C} 25 \mathrm{~A}$ & $0.0970(17)$ & $0.147(2)$ & $0.0718(14)$ & $-0.0226(16)$ & $-0.0119(12)$ & $0.0078(14)$ \\
\hline F1B & $0.1881(15)$ & $0.0904(9)$ & $0.0925(9)$ & $-0.0023(10)$ & $0.0239(9)$ & $0.0314(7)$ \\
\hline O1B & $0.0677(8)$ & $0.0835(9)$ & $0.0804(8)$ & $-0.0159(7)$ & $-0.0044(7)$ & $0.0024(7)$ \\
\hline $\mathrm{O} 2 \mathrm{~B}$ & $0.0885(9)$ & $0.0646(8)$ & $0.0843(9)$ & $0.0185(7)$ & $0.0103(7)$ & $-0.0045(6)$ \\
\hline N1B & $0.0605(8)$ & $0.0512(8)$ & $0.0615(8)$ & $0.0053(7)$ & $0.0018(7)$ & $0.0024(6)$ \\
\hline $\mathrm{N} 2 \mathrm{~B}$ & $0.0564(8)$ & $0.0535(8)$ & $0.0558(7)$ & $0.0056(7)$ & $-0.0021(6)$ & $-0.0003(6)$ \\
\hline $\mathrm{C} 1 \mathrm{~B}$ & $0.1186(18)$ & $0.0606(12)$ & $0.0675(12)$ & $0.0130(13)$ & $0.0217(12)$ & $0.0125(10)$ \\
\hline $\mathrm{C} 2 \mathrm{~B}$ & $0.1173(18)$ & $0.0800(15)$ & $0.0631(12)$ & $0.0123(14)$ & $-0.0109(12)$ & $0.0060(11)$ \\
\hline $\mathrm{C} 3 \mathrm{~B}$ & $0.0839(13)$ & $0.0732(12)$ & $0.0685(11)$ & $0.0027(11)$ & $-0.0118(10)$ & $-0.0017(10)$ \\
\hline C4B & $0.0579(10)$ & $0.0538(10)$ & $0.0558(9)$ & $0.0083(8)$ & $0.0043(8)$ & $-0.0035(8)$ \\
\hline C5B & $0.0781(13)$ & $0.0672(12)$ & $0.0667(11)$ & $-0.0060(10)$ & $-0.0023(9)$ & $0.0029(9)$ \\
\hline C6B & $0.0966(15)$ & $0.0675(12)$ & $0.0861(14)$ & $-0.0106(11)$ & $0.0060(12)$ & $0.0074(11)$ \\
\hline C7B & $0.0521(10)$ & $0.0572(10)$ & $0.0616(9)$ & $0.0049(9)$ & $0.0050(8)$ & $-0.0062(8)$ \\
\hline $\mathrm{C} 8 \mathrm{~B}$ & $0.0610(10)$ & $0.0523(10)$ & $0.0688(10)$ & $0.0017(8)$ & $-0.0030(8)$ & $0.0033(8)$ \\
\hline C9B & $0.0604(11)$ & $0.0611(11)$ & $0.0586(9)$ & 0.0077 (9) & $-0.0038(8)$ & $-0.0063(8)$ \\
\hline C10B & $0.0531(9)$ & $0.0658(11)$ & $0.0500(8)$ & $0.0006(8)$ & $-0.0049(7)$ & $-0.0051(8)$ \\
\hline C11B & $0.0561(10)$ & $0.0578(10)$ & $0.0537(9)$ & $-0.0020(8)$ & $-0.0030(7)$ & $0.0012(7)$ \\
\hline $\mathrm{C} 12 \mathrm{~B}$ & $0.0527(9)$ & $0.0532(9)$ & $0.0484(8)$ & $-0.0009(8)$ & $-0.0059(7)$ & $-0.0011(7)$ \\
\hline C13B & $0.0546(9)$ & $0.0530(9)$ & $0.0505(8)$ & $-0.0001(8)$ & $-0.0078(7)$ & $-0.0035(7)$ \\
\hline C14B & $0.0657(11)$ & $0.0610(10)$ & $0.0565(9)$ & $0.0048(9)$ & $0.0023(8)$ & $0.0004(8)$ \\
\hline C15B & $0.0708(12)$ & $0.0685(12)$ & $0.0660(10)$ & $0.0085(10)$ & $0.0074(9)$ & $-0.0053(9)$ \\
\hline C16B & $0.0672(12)$ & $0.0640(11)$ & $0.0738(11)$ & $0.0137(9)$ & $-0.0071(9)$ & $-0.0103(9)$ \\
\hline C17B & $0.0788(12)$ & $0.0541(10)$ & $0.0721(11)$ & $0.0045(9)$ & $-0.0055(10)$ & $0.0042(8)$ \\
\hline C18B & $0.0653(11)$ & $0.0602(11)$ & $0.0653(10)$ & $0.0023(9)$ & $0.0041(8)$ & $0.0031(8)$ \\
\hline C19B & $0.0555(10)$ & $0.0838(13)$ & $0.0593(10)$ & $0.0040(9)$ & $0.0005(8)$ & $-0.0065(9)$ \\
\hline $\mathrm{C} 20 \mathrm{~B}$ & $0.0521(9)$ & $0.0577(10)$ & $0.0571(9)$ & $0.0021(8)$ & $0.0056(8)$ & $-0.0074(8)$ \\
\hline C21B & $0.1225(19)$ & $0.0981(16)$ & $0.0714(13)$ & $-0.0528(15)$ & $-0.0054(12)$ & $-0.0004(11)$ \\
\hline $\mathrm{C} 22 \mathrm{~B}$ & $0.166(3)$ & $0.0959(17)$ & $0.0681(13)$ & $-0.0511(18)$ & $-0.0095(14)$ & $0.0116(12)$ \\
\hline $\mathrm{C} 23 \mathrm{~B}$ & $0.1031(16)$ & $0.0903(15)$ & $0.0592(11)$ & $0.0007(13)$ & $-0.0068(11)$ & $-0.0107(11)$ \\
\hline $\mathrm{C} 24 \mathrm{~B}$ & $0.129(2)$ & $0.158(2)$ & $0.0696(14)$ & $-0.0650(19)$ & $-0.0042(13)$ & $-0.0204(15)$ \\
\hline $\mathrm{C} 25 \mathrm{~B}$ & $0.135(2)$ & $0.134(2)$ & $0.0635(13)$ & $-0.0703(18)$ & $0.0033(13)$ & $-0.0048(13)$ \\
\hline
\end{tabular}

Geometric parameters $\left(\AA,{ }^{\circ}\right)$

\begin{tabular}{llll}
\hline $\mathrm{F} 1 \mathrm{~A}-\mathrm{C} 1 \mathrm{~A}$ & $1.348(3)$ & $\mathrm{F} 1 \mathrm{~B}-\mathrm{C} 1 \mathrm{~B}$ & $1.353(2)$ \\
$\mathrm{O} 1 \mathrm{~A}-\mathrm{C} 7 \mathrm{~A}$ & $1.215(2)$ & $\mathrm{O} 1 \mathrm{~B}-\mathrm{C} 7 \mathrm{~B}$ & $1.217(2)$ \\
$\mathrm{O} 2 \mathrm{~A}-\mathrm{C} 9 \mathrm{~A}$ & $1.229(2)$ & $\mathrm{O} 2 \mathrm{~B}-\mathrm{C} 9 \mathrm{~B}$ & $1.232(2)$ \\
$\mathrm{N} 1 \mathrm{~A}-\mathrm{N} 2 \mathrm{~A}$ & $1.353(2)$ & $\mathrm{N} 1 \mathrm{~B}-\mathrm{N} 2 \mathrm{~B}$ & $1.3532(17)$ \\
$\mathrm{N} 1 \mathrm{~A}-\mathrm{C} 9 \mathrm{~A}$ & $1.384(2)$ & $\mathrm{N} 1 \mathrm{~B}-\mathrm{C} 9 \mathrm{~B}$ & $1.449(2)$ \\
$\mathrm{N} 1 \mathrm{~A}-\mathrm{C} 8 \mathrm{~A}$ & $1.450(2)$ & $\mathrm{N} 1 \mathrm{~B}-\mathrm{C} 8 \mathrm{~B}$ & $1.310(2)$ \\
$\mathrm{N} 2 \mathrm{~A}-\mathrm{C} 12 \mathrm{~A}$ & $1.309(2)$ & $\mathrm{N} 2 \mathrm{~B}-\mathrm{C} 12 \mathrm{~B}$ & $1.354(3)$ \\
$\mathrm{C} 1 \mathrm{~A}-\mathrm{C} 2 \mathrm{~A}$ & $1.349(4)$ & $\mathrm{C} 1 \mathrm{~B}-\mathrm{C} 2 \mathrm{~B}$ & $1.366(3)$ \\
$\mathrm{C} 1 \mathrm{~A}-\mathrm{C} 6 \mathrm{~A}$ & $1.353(4)$ & $\mathrm{C} 1 \mathrm{~B}-\mathrm{C} 6 \mathrm{~B}$ & $1.369(3)$ \\
$\mathrm{C} 2 \mathrm{~A}-\mathrm{C} 3 \mathrm{~A}$ & $1.373(3)$ & $\mathrm{C} 2 \mathrm{~B}-\mathrm{C} 3 \mathrm{~B}$ & 0.9300 \\
$\mathrm{C} 2 \mathrm{~A}-\mathrm{H} 2 \mathrm{~A}$ & 0.9300 & $\mathrm{C} 2 \mathrm{~B}-\mathrm{H} 2 \mathrm{~B}$ &
\end{tabular}




\begin{tabular}{|c|c|c|c|}
\hline $\mathrm{C} 3 \mathrm{~A}-\mathrm{C} 4 \mathrm{~A}$ & $1.389(3)$ & $\mathrm{C} 3 \mathrm{~B}-\mathrm{C} 4 \mathrm{~B}$ & $1.389(2)$ \\
\hline $\mathrm{C} 3 \mathrm{~A}-\mathrm{H} 3 \mathrm{~A}$ & 0.9300 & $\mathrm{C} 3 \mathrm{~B}-\mathrm{H} 3 \mathrm{~B}$ & 0.9300 \\
\hline $\mathrm{C} 4 \mathrm{~A}-\mathrm{C} 5 \mathrm{~A}$ & $1.375(3)$ & $\mathrm{C} 4 \mathrm{~B}-\mathrm{C} 5 \mathrm{~B}$ & $1.378(3)$ \\
\hline $\mathrm{C} 4 \mathrm{~A}-\mathrm{C} 7 \mathrm{~A}$ & $1.476(3)$ & $\mathrm{C} 4 \mathrm{~B}-\mathrm{C} 7 \mathrm{~B}$ & $1.483(2)$ \\
\hline $\mathrm{C} 5 \mathrm{~A}-\mathrm{C} 6 \mathrm{~A}$ & $1.382(3)$ & $\mathrm{C} 5 \mathrm{~B}-\mathrm{C} 6 \mathrm{~B}$ & $1.384(3)$ \\
\hline $\mathrm{C} 5 \mathrm{~A}-\mathrm{H} 5 \mathrm{~A}$ & 0.9300 & $\mathrm{C} 5 \mathrm{~B}-\mathrm{H} 5 \mathrm{~B}$ & 0.9300 \\
\hline C6A-H6A & 0.9300 & $\mathrm{C} 6 \mathrm{~B}-\mathrm{H} 6 \mathrm{~B}$ & 0.9300 \\
\hline $\mathrm{C} 7 \mathrm{~A}-\mathrm{C} 8 \mathrm{~A}$ & $1.512(3)$ & $\mathrm{C} 7 \mathrm{~B}-\mathrm{C} 8 \mathrm{~B}$ & $1.517(2)$ \\
\hline $\mathrm{C} 8 \mathrm{~A}-\mathrm{H} 8 \mathrm{~A}$ & 0.9700 & $\mathrm{C} 8 \mathrm{~B}-\mathrm{H} 8 \mathrm{C}$ & 0.9700 \\
\hline $\mathrm{C} 8 \mathrm{~A}-\mathrm{H} 8 \mathrm{~B}$ & 0.9700 & $\mathrm{C} 8 \mathrm{~B}-\mathrm{H} 8 \mathrm{D}$ & 0.9700 \\
\hline $\mathrm{C} 9 \mathrm{~A}-\mathrm{C} 10 \mathrm{~A}$ & $1.445(3)$ & $\mathrm{C} 9 \mathrm{~B}-\mathrm{C} 10 \mathrm{~B}$ & $1.449(2)$ \\
\hline $\mathrm{C} 10 \mathrm{~A}-\mathrm{C} 11 \mathrm{~A}$ & $1.342(2)$ & $\mathrm{C} 10 \mathrm{~B}-\mathrm{C} 11 \mathrm{~B}$ & $1.344(2)$ \\
\hline $\mathrm{C} 10 \mathrm{~A}-\mathrm{C} 19 \mathrm{~A}$ & $1.511(3)$ & $\mathrm{C} 10 \mathrm{~B}-\mathrm{C} 19 \mathrm{~B}$ & $1.505(2)$ \\
\hline $\mathrm{C} 11 \mathrm{~A}-\mathrm{C} 12 \mathrm{~A}$ & $1.430(2)$ & $\mathrm{C} 11 \mathrm{~B}-\mathrm{C} 12 \mathrm{~B}$ & $1.427(2)$ \\
\hline $\mathrm{C} 11 \mathrm{~A}-\mathrm{H} 11 \mathrm{~A}$ & 0.9300 & $\mathrm{C} 11 \mathrm{~B}-\mathrm{H} 11 \mathrm{~B}$ & 0.9300 \\
\hline $\mathrm{C} 12 \mathrm{~A}-\mathrm{C} 13 \mathrm{~A}$ & $1.486(2)$ & $\mathrm{C} 12 \mathrm{~B}-\mathrm{C} 13 \mathrm{~B}$ & $1.485(2)$ \\
\hline $\mathrm{C} 13 \mathrm{~A}-\mathrm{C} 18 \mathrm{~A}$ & $1.386(3)$ & $\mathrm{C} 13 \mathrm{~B}-\mathrm{C} 18 \mathrm{~B}$ & $1.388(2)$ \\
\hline $\mathrm{C} 13 \mathrm{~A}-\mathrm{C} 14 \mathrm{~A}$ & $1.391(2)$ & $\mathrm{C} 13 \mathrm{~B}-\mathrm{C} 14 \mathrm{~B}$ & $1.391(2)$ \\
\hline $\mathrm{C} 14 \mathrm{~A}-\mathrm{C} 15 \mathrm{~A}$ & $1.379(3)$ & $\mathrm{C} 14 \mathrm{~B}-\mathrm{C} 15 \mathrm{~B}$ & $1.374(2)$ \\
\hline $\mathrm{C} 14 \mathrm{~A}-\mathrm{H} 14 \mathrm{~A}$ & 0.9300 & $\mathrm{C} 14 \mathrm{~B}-\mathrm{H} 14 \mathrm{~B}$ & 0.9300 \\
\hline $\mathrm{C} 15 \mathrm{~A}-\mathrm{C} 16 \mathrm{~A}$ & $1.372(3)$ & $\mathrm{C} 15 \mathrm{~B}-\mathrm{C} 16 \mathrm{~B}$ & $1.376(3)$ \\
\hline $\mathrm{C} 15 \mathrm{~A}-\mathrm{H} 15 \mathrm{~A}$ & 0.9300 & $\mathrm{C} 15 \mathrm{~B}-\mathrm{H} 15 \mathrm{~B}$ & 0.9300 \\
\hline $\mathrm{C} 16 \mathrm{~A}-\mathrm{C} 17 \mathrm{~A}$ & $1.363(3)$ & $\mathrm{C} 16 \mathrm{~B}-\mathrm{C} 17 \mathrm{~B}$ & $1.365(3)$ \\
\hline $\mathrm{C} 16 \mathrm{~A}-\mathrm{H} 16 \mathrm{~A}$ & 0.9300 & $\mathrm{C} 16 \mathrm{~B}-\mathrm{H} 16 \mathrm{~B}$ & 0.9300 \\
\hline $\mathrm{C} 17 \mathrm{~A}-\mathrm{C} 18 \mathrm{~A}$ & $1.383(3)$ & $\mathrm{C} 17 \mathrm{~B}-\mathrm{C} 18 \mathrm{~B}$ & $1.383(2)$ \\
\hline $\mathrm{C} 17 \mathrm{~A}-\mathrm{H} 17 \mathrm{~A}$ & 0.9300 & $\mathrm{C} 17 \mathrm{~B}-\mathrm{H} 17 \mathrm{~B}$ & 0.9300 \\
\hline C18A-H18A & 0.9300 & $\mathrm{C} 18 \mathrm{~B}-\mathrm{H} 18 \mathrm{~B}$ & 0.9300 \\
\hline $\mathrm{C} 19 \mathrm{~A}-\mathrm{C} 20 \mathrm{~A}$ & $1.498(3)$ & $\mathrm{C} 19 \mathrm{~B}-\mathrm{C} 20 \mathrm{~B}$ & $1.505(2)$ \\
\hline C19A-H19A & 0.9700 & $\mathrm{C} 19 \mathrm{~B}-\mathrm{H} 19 \mathrm{C}$ & 0.9700 \\
\hline C19A-H19B & 0.9700 & $\mathrm{C} 19 \mathrm{~B}-\mathrm{H} 19 \mathrm{D}$ & 0.9700 \\
\hline $\mathrm{C} 20 \mathrm{~A}-\mathrm{C} 21 \mathrm{~A}$ & $1.356(3)$ & $\mathrm{C} 20 \mathrm{~B}-\mathrm{C} 21 \mathrm{~B}$ & $1.356(3)$ \\
\hline $\mathrm{C} 20 \mathrm{~A}-\mathrm{C} 25 \mathrm{~A}$ & $1.379(3)$ & $\mathrm{C} 20 \mathrm{~B}-\mathrm{C} 25 \mathrm{~B}$ & $1.363(3)$ \\
\hline $\mathrm{C} 21 \mathrm{~A}-\mathrm{C} 22 \mathrm{~A}$ & $1.416(5)$ & $\mathrm{C} 21 \mathrm{~B}-\mathrm{C} 22 \mathrm{~B}$ & $1.391(3)$ \\
\hline $\mathrm{C} 21 \mathrm{~A}-\mathrm{H} 21 \mathrm{~A}$ & 0.9300 & $\mathrm{C} 21 \mathrm{~B}-\mathrm{H} 21 \mathrm{~B}$ & 0.9300 \\
\hline $\mathrm{C} 22 \mathrm{~A}-\mathrm{C} 23 \mathrm{~A}$ & $1.375(5)$ & $\mathrm{C} 22 \mathrm{~B}-\mathrm{C} 23 \mathrm{~B}$ & $1.320(3)$ \\
\hline $\mathrm{C} 22 \mathrm{~A}-\mathrm{H} 22 \mathrm{~A}$ & 0.9300 & $\mathrm{C} 22 \mathrm{~B}-\mathrm{H} 22 \mathrm{~B}$ & 0.9300 \\
\hline $\mathrm{C} 23 \mathrm{~A}-\mathrm{C} 24 \mathrm{~A}$ & $1.329(6)$ & $\mathrm{C} 23 \mathrm{~B}-\mathrm{C} 24 \mathrm{~B}$ & $1.342(3)$ \\
\hline $\mathrm{C} 23 \mathrm{~A}-\mathrm{H} 23 \mathrm{~A}$ & 0.9300 & $\mathrm{C} 23 \mathrm{~B}-\mathrm{H} 23 \mathrm{~B}$ & 0.9300 \\
\hline $\mathrm{C} 24 \mathrm{~A}-\mathrm{C} 25 \mathrm{~A}$ & $1.354(4)$ & $\mathrm{C} 24 \mathrm{~B}-\mathrm{C} 25 \mathrm{~B}$ & $1.378(3)$ \\
\hline $\mathrm{C} 24 \mathrm{~A}-\mathrm{H} 24$ & 0.9300 & $\mathrm{C} 24 \mathrm{~B}-\mathrm{H} 24 \mathrm{~B}$ & 0.9300 \\
\hline $\mathrm{C} 25 \mathrm{~A}-\mathrm{H} 25 \mathrm{~A}$ & 0.9300 & $\mathrm{C} 25 \mathrm{~B}-\mathrm{H} 25 \mathrm{~B}$ & 0.9300 \\
\hline $\mathrm{N} 2 \mathrm{~A}-\mathrm{N} 1 \mathrm{~A}-\mathrm{C} 9 \mathrm{~A}$ & $126.89(15)$ & $\mathrm{N} 2 \mathrm{~B}-\mathrm{N} 1 \mathrm{~B}-\mathrm{C} 9 \mathrm{~B}$ & $126.71(14)$ \\
\hline $\mathrm{N} 2 \mathrm{~A}-\mathrm{N} 1 \mathrm{~A}-\mathrm{C} 8 \mathrm{~A}$ & $114.62(14)$ & $\mathrm{N} 2 \mathrm{~B}-\mathrm{N} 1 \mathrm{~B}-\mathrm{C} 8 \mathrm{~B}$ & $113.92(13)$ \\
\hline $\mathrm{C} 9 \mathrm{~A}-\mathrm{N} 1 \mathrm{~A}-\mathrm{C} 8 \mathrm{~A}$ & $118.45(16)$ & $\mathrm{C} 9 \mathrm{~B}-\mathrm{N} 1 \mathrm{~B}-\mathrm{C} 8 \mathrm{~B}$ & $119.37(13)$ \\
\hline $\mathrm{C} 12 \mathrm{~A}-\mathrm{N} 2 \mathrm{~A}-\mathrm{N} 1 \mathrm{~A}$ & $117.72(14)$ & $\mathrm{C} 12 \mathrm{~B}-\mathrm{N} 2 \mathrm{~B}-\mathrm{N} 1 \mathrm{~B}$ & $117.52(13)$ \\
\hline $\mathrm{F} 1 \mathrm{~A}-\mathrm{C} 1 \mathrm{~A}-\mathrm{C} 2 \mathrm{~A}$ & $118.3(3)$ & $\mathrm{F} 1 \mathrm{~B}-\mathrm{C} 1 \mathrm{~B}-\mathrm{C} 2 \mathrm{~B}$ & $119.3(2)$ \\
\hline $\mathrm{F} 1 \mathrm{~A}-\mathrm{C} 1 \mathrm{~A}-\mathrm{C} 6 \mathrm{~A}$ & $118.9(3)$ & $\mathrm{F} 1 \mathrm{~B}-\mathrm{C} 1 \mathrm{~B}-\mathrm{C} 6 \mathrm{~B}$ & $117.6(2)$ \\
\hline
\end{tabular}




\begin{tabular}{|c|c|}
\hline $\mathrm{C} 2 \mathrm{~A}-\mathrm{C} 1 \mathrm{~A}-\mathrm{C} 6 \mathrm{~A}$ & $122.9(2)$ \\
\hline $\mathrm{C} 1 \mathrm{~A}-\mathrm{C} 2 \mathrm{~A}-\mathrm{C} 3 \mathrm{~A}$ & $118.2(2)$ \\
\hline $\mathrm{C} 1 \mathrm{~A}-\mathrm{C} 2 \mathrm{~A}-\mathrm{H} 2 \mathrm{~A}$ & 120.9 \\
\hline $\mathrm{C} 3 \mathrm{~A}-\mathrm{C} 2 \mathrm{~A}-\mathrm{H} 2 \mathrm{~A}$ & 120.9 \\
\hline $\mathrm{C} 2 \mathrm{~A}-\mathrm{C} 3 \mathrm{~A}-\mathrm{C} 4 \mathrm{~A}$ & $121.5(2)$ \\
\hline $\mathrm{C} 2 \mathrm{~A}-\mathrm{C} 3 \mathrm{~A}-\mathrm{H} 3 \mathrm{~A}$ & 119.2 \\
\hline $\mathrm{C} 4 \mathrm{~A}-\mathrm{C} 3 \mathrm{~A}-\mathrm{H} 3 \mathrm{~A}$ & 119.2 \\
\hline $\mathrm{C} 5 \mathrm{~A}-\mathrm{C} 4 \mathrm{~A}-\mathrm{C} 3 \mathrm{~A}$ & $117.84(19)$ \\
\hline $\mathrm{C} 5 \mathrm{~A}-\mathrm{C} 4 \mathrm{~A}-\mathrm{C} 7 \mathrm{~A}$ & $122.41(18)$ \\
\hline $\mathrm{C} 3 \mathrm{~A}-\mathrm{C} 4 \mathrm{~A}-\mathrm{C} 7 \mathrm{~A}$ & $119.72(19)$ \\
\hline $\mathrm{C} 4 \mathrm{~A}-\mathrm{C} 5 \mathrm{~A}-\mathrm{C} 6 \mathrm{~A}$ & $120.9(2)$ \\
\hline $\mathrm{C} 4 \mathrm{~A}-\mathrm{C} 5 \mathrm{~A}-\mathrm{H} 5 \mathrm{~A}$ & 119.6 \\
\hline $\mathrm{C} 6 \mathrm{~A}-\mathrm{C} 5 \mathrm{~A}-\mathrm{H} 5 \mathrm{~A}$ & 119.6 \\
\hline $\mathrm{C} 1 \mathrm{~A}-\mathrm{C} 6 \mathrm{~A}-\mathrm{C} 5 \mathrm{~A}$ & $118.7(3)$ \\
\hline $\mathrm{C} 1 \mathrm{~A}-\mathrm{C} 6 \mathrm{~A}-\mathrm{H} 6 \mathrm{~A}$ & 120.6 \\
\hline $\mathrm{C} 5 \mathrm{~A}-\mathrm{C} 6 \mathrm{~A}-\mathrm{H} 6 \mathrm{~A}$ & 120.6 \\
\hline $\mathrm{O} 1 \mathrm{~A}-\mathrm{C} 7 \mathrm{~A}-\mathrm{C} 4 \mathrm{~A}$ & $121.70(18)$ \\
\hline $\mathrm{O} 1 \mathrm{~A}-\mathrm{C} 7 \mathrm{~A}-\mathrm{C} 8 \mathrm{~A}$ & $121.01(17)$ \\
\hline $\mathrm{C} 4 \mathrm{~A}-\mathrm{C} 7 \mathrm{~A}-\mathrm{C} 8 \mathrm{~A}$ & $117.26(17)$ \\
\hline $\mathrm{N} 1 \mathrm{~A}-\mathrm{C} 8 \mathrm{~A}-\mathrm{C} 7 \mathrm{~A}$ & $113.57(16)$ \\
\hline $\mathrm{N} 1 \mathrm{~A}-\mathrm{C} 8 \mathrm{~A}-\mathrm{H} 8 \mathrm{~A}$ & 108.9 \\
\hline $\mathrm{C} 7 \mathrm{~A}-\mathrm{C} 8 \mathrm{~A}-\mathrm{H} 8 \mathrm{~A}$ & 108.9 \\
\hline $\mathrm{N} 1 \mathrm{~A}-\mathrm{C} 8 \mathrm{~A}-\mathrm{H} 8 \mathrm{~B}$ & 108.9 \\
\hline $\mathrm{C} 7 \mathrm{~A}-\mathrm{C} 8 \mathrm{~A}-\mathrm{H} 8 \mathrm{~B}$ & 108.9 \\
\hline $\mathrm{H} 8 \mathrm{~A}-\mathrm{C} 8 \mathrm{~A}-\mathrm{H} 8 \mathrm{~B}$ & 107.7 \\
\hline $\mathrm{O} 2 \mathrm{~A}-\mathrm{C} 9 \mathrm{~A}-\mathrm{N} 1 \mathrm{~A}$ & $120.46(17)$ \\
\hline $\mathrm{O} 2 \mathrm{~A}-\mathrm{C} 9 \mathrm{~A}-\mathrm{C} 10 \mathrm{~A}$ & $125.55(18)$ \\
\hline $\mathrm{N} 1 \mathrm{~A}-\mathrm{C} 9 \mathrm{~A}-\mathrm{C} 10 \mathrm{~A}$ & $113.95(17)$ \\
\hline $\mathrm{C} 11 \mathrm{~A}-\mathrm{C} 10 \mathrm{~A}-\mathrm{C} 9 \mathrm{~A}$ & $119.16(16)$ \\
\hline $\mathrm{C} 11 \mathrm{~A}-\mathrm{C} 10 \mathrm{~A}-\mathrm{C} 19 \mathrm{~A}$ & $124.93(17)$ \\
\hline $\mathrm{C} 9 \mathrm{~A}-\mathrm{C} 10 \mathrm{~A}-\mathrm{C} 19 \mathrm{~A}$ & $115.91(17)$ \\
\hline $\mathrm{C} 10 \mathrm{~A}-\mathrm{C} 11 \mathrm{~A}-\mathrm{C} 12 \mathrm{~A}$ & $121.47(17)$ \\
\hline $\mathrm{C} 10 \mathrm{~A}-\mathrm{C} 11 \mathrm{~A}-\mathrm{H} 11 \mathrm{~A}$ & 119.3 \\
\hline $\mathrm{C} 12 \mathrm{~A}-\mathrm{C} 11 \mathrm{~A}-\mathrm{H} 11 \mathrm{~A}$ & 119.3 \\
\hline $\mathrm{N} 2 \mathrm{~A}-\mathrm{C} 12 \mathrm{~A}-\mathrm{C} 11 \mathrm{~A}$ & $120.52(17)$ \\
\hline $\mathrm{N} 2 \mathrm{~A}-\mathrm{C} 12 \mathrm{~A}-\mathrm{C} 13 \mathrm{~A}$ & $115.78(15)$ \\
\hline $\mathrm{C} 11 \mathrm{~A}-\mathrm{C} 12 \mathrm{~A}-\mathrm{C} 13 \mathrm{~A}$ & $123.59(16)$ \\
\hline $\mathrm{C} 18 \mathrm{~A}-\mathrm{C} 13 \mathrm{~A}-\mathrm{C} 14 \mathrm{~A}$ & $117.33(17)$ \\
\hline $\mathrm{C} 18 \mathrm{~A}-\mathrm{C} 13 \mathrm{~A}-\mathrm{C} 12 \mathrm{~A}$ & $121.94(16)$ \\
\hline $\mathrm{C} 14 \mathrm{~A}-\mathrm{C} 13 \mathrm{~A}-\mathrm{C} 12 \mathrm{~A}$ & $120.70(16)$ \\
\hline $\mathrm{C} 15 \mathrm{~A}-\mathrm{C} 14 \mathrm{~A}-\mathrm{C} 13 \mathrm{~A}$ & $120.89(18)$ \\
\hline $\mathrm{C} 15 \mathrm{~A}-\mathrm{C} 14 \mathrm{~A}-\mathrm{H} 14 \mathrm{~A}$ & 119.6 \\
\hline $\mathrm{C} 13 \mathrm{~A}-\mathrm{C} 14 \mathrm{~A}-\mathrm{H} 14 \mathrm{~A}$ & 119.6 \\
\hline $\mathrm{C} 16 \mathrm{~A}-\mathrm{C} 15 \mathrm{~A}-\mathrm{C} 14 \mathrm{~A}$ & $120.77(18)$ \\
\hline $\mathrm{C} 16 \mathrm{~A}-\mathrm{C} 15 \mathrm{~A}-\mathrm{H} 15 \mathrm{~A}$ & 119.6 \\
\hline $\mathrm{C} 14 \mathrm{~A}-\mathrm{C} 15 \mathrm{~A}-\mathrm{H} 15 \mathrm{~A}$ & 119.6 \\
\hline $\mathrm{C} 17 \mathrm{~A}-\mathrm{C} 16 \mathrm{~A}-\mathrm{C} 15 \mathrm{~A}$ & $119.18(19)$ \\
\hline & \\
\hline
\end{tabular}

$123.07(19)$

$118.2(2)$

120.9

120.9

121.3 (2)

119.3

119.3

$118.68(17)$

$122.54(16)$

$118.75(17)$

$120.48(19)$

119.8

119.8

$118.3(2)$

120.9

120.9

$121.68(16)$

$120.65(16)$

$117.65(16)$

$112.24(15)$

109.2

109.2

109.2

109.2

107.9

$120.15(16)$

$125.22(16)$

$114.63(14)$

$118.57(15)$

122.85 (16)

$118.58(15)$

$121.60(15)$

119.2

119.2

120.95 (14)

115.81 (14)

$123.17(14)$

$117.53(15)$

121.44 (14)

120.96 (14)

121.11 (16)

119.4

119.4

120.31 (17)

119.8

119.8

119.71 (17)

120.1 


\begin{tabular}{|c|c|c|c|}
\hline $\mathrm{C} 15 \mathrm{~A}-\mathrm{C} 16 \mathrm{~A}-\mathrm{H} 16 \mathrm{~A}$ & 120.4 & $\mathrm{C} 15 \mathrm{~B}-\mathrm{C} 16 \mathrm{~B}-\mathrm{H} 16 \mathrm{~B}$ & 120.1 \\
\hline $\mathrm{C} 16 \mathrm{~A}-\mathrm{C} 17 \mathrm{~A}-\mathrm{C} 18 \mathrm{~A}$ & $120.60(19)$ & $\mathrm{C} 16 \mathrm{~B}-\mathrm{C} 17 \mathrm{~B}-\mathrm{C} 18 \mathrm{~B}$ & $120.22(17)$ \\
\hline $\mathrm{C} 16 \mathrm{~A}-\mathrm{C} 17 \mathrm{~A}-\mathrm{H} 17 \mathrm{~A}$ & 119.7 & $\mathrm{C} 16 \mathrm{~B}-\mathrm{C} 17 \mathrm{~B}-\mathrm{H} 17 \mathrm{~B}$ & 119.9 \\
\hline $\mathrm{C} 18 \mathrm{~A}-\mathrm{C} 17 \mathrm{~A}-\mathrm{H} 17 \mathrm{~A}$ & 119.7 & $\mathrm{C} 18 \mathrm{~B}-\mathrm{C} 17 \mathrm{~B}-\mathrm{H} 17 \mathrm{~B}$ & 119.9 \\
\hline $\mathrm{C} 17 \mathrm{~A}-\mathrm{C} 18 \mathrm{~A}-\mathrm{C} 13 \mathrm{~A}$ & $121.23(18)$ & $\mathrm{C} 17 \mathrm{~B}-\mathrm{C} 18 \mathrm{~B}-\mathrm{C} 13 \mathrm{~B}$ & $121.11(16)$ \\
\hline $\mathrm{C} 17 \mathrm{~A}-\mathrm{C} 18 \mathrm{~A}-\mathrm{H} 18 \mathrm{~A}$ & 119.4 & $\mathrm{C} 17 \mathrm{~B}-\mathrm{C} 18 \mathrm{~B}-\mathrm{H} 18 \mathrm{~B}$ & 119.4 \\
\hline $\mathrm{C} 13 \mathrm{~A}-\mathrm{C} 18 \mathrm{~A}-\mathrm{H} 18 \mathrm{~A}$ & 119.4 & $\mathrm{C} 13 \mathrm{~B}-\mathrm{C} 18 \mathrm{~B}-\mathrm{H} 18 \mathrm{~B}$ & 119.4 \\
\hline $\mathrm{C} 20 \mathrm{~A}-\mathrm{C} 19 \mathrm{~A}-\mathrm{C} 10 \mathrm{~A}$ & $114.86(17)$ & $\mathrm{C} 10 \mathrm{~B}-\mathrm{C} 19 \mathrm{~B}-\mathrm{C} 20 \mathrm{~B}$ & $113.81(14)$ \\
\hline $\mathrm{C} 20 \mathrm{~A}-\mathrm{C} 19 \mathrm{~A}-\mathrm{H} 19 \mathrm{~A}$ & 108.6 & $\mathrm{C} 10 \mathrm{~B}-\mathrm{C} 19 \mathrm{~B}-\mathrm{H} 19 \mathrm{C}$ & 108.8 \\
\hline $\mathrm{C} 10 \mathrm{~A}-\mathrm{C} 19 \mathrm{~A}-\mathrm{H} 19 \mathrm{~A}$ & 108.6 & $\mathrm{C} 20 \mathrm{~B}-\mathrm{C} 19 \mathrm{~B}-\mathrm{H} 19 \mathrm{C}$ & 108.8 \\
\hline $\mathrm{C} 20 \mathrm{~A}-\mathrm{C} 19 \mathrm{~A}-\mathrm{H} 19 \mathrm{~B}$ & 108.6 & $\mathrm{C} 10 \mathrm{~B}-\mathrm{C} 19 \mathrm{~B}-\mathrm{H} 19 \mathrm{D}$ & 108.8 \\
\hline $\mathrm{C} 10 \mathrm{~A}-\mathrm{C} 19 \mathrm{~A}-\mathrm{H} 19 \mathrm{~B}$ & 108.6 & $\mathrm{C} 20 \mathrm{~B}-\mathrm{C} 19 \mathrm{~B}-\mathrm{H} 19 \mathrm{D}$ & 108.8 \\
\hline $\mathrm{H} 19 \mathrm{~A}-\mathrm{C} 19 \mathrm{~A}-\mathrm{H} 19 \mathrm{~B}$ & 107.5 & $\mathrm{H} 19 \mathrm{C}-\mathrm{C} 19 \mathrm{~B}-\mathrm{H} 19 \mathrm{D}$ & 107.7 \\
\hline $\mathrm{C} 21 \mathrm{~A}-\mathrm{C} 20 \mathrm{~A}-\mathrm{C} 25 \mathrm{~A}$ & $117.7(2)$ & $\mathrm{C} 21 \mathrm{~B}-\mathrm{C} 20 \mathrm{~B}-\mathrm{C} 25 \mathrm{~B}$ & $115.85(18)$ \\
\hline $\mathrm{C} 21 \mathrm{~A}-\mathrm{C} 20 \mathrm{~A}-\mathrm{C} 19 \mathrm{~A}$ & $122.8(2)$ & $\mathrm{C} 21 \mathrm{~B}-\mathrm{C} 20 \mathrm{~B}-\mathrm{C} 19 \mathrm{~B}$ & $121.93(17)$ \\
\hline $\mathrm{C} 25 \mathrm{~A}-\mathrm{C} 20 \mathrm{~A}-\mathrm{C} 19 \mathrm{~A}$ & $119.5(2)$ & $\mathrm{C} 25 \mathrm{~B}-\mathrm{C} 20 \mathrm{~B}-\mathrm{C} 19 \mathrm{~B}$ & $122.22(17)$ \\
\hline $\mathrm{C} 20 \mathrm{~A}-\mathrm{C} 21 \mathrm{~A}-\mathrm{C} 22 \mathrm{~A}$ & $120.1(3)$ & $\mathrm{C} 20 \mathrm{~B}-\mathrm{C} 21 \mathrm{~B}-\mathrm{C} 22 \mathrm{~B}$ & $121.4(2)$ \\
\hline $\mathrm{C} 20 \mathrm{~A}-\mathrm{C} 21 \mathrm{~A}-\mathrm{H} 21 \mathrm{~A}$ & 120.0 & $\mathrm{C} 20 \mathrm{~B}-\mathrm{C} 21 \mathrm{~B}-\mathrm{H} 21 \mathrm{~B}$ & 119.3 \\
\hline $\mathrm{C} 22 \mathrm{~A}-\mathrm{C} 21 \mathrm{~A}-\mathrm{H} 21 \mathrm{~A}$ & 120.0 & $\mathrm{C} 22 \mathrm{~B}-\mathrm{C} 21 \mathrm{~B}-\mathrm{H} 21 \mathrm{~B}$ & 119.3 \\
\hline $\mathrm{C} 23 \mathrm{~A}-\mathrm{C} 22 \mathrm{~A}-\mathrm{C} 21 \mathrm{~A}$ & $119.1(4)$ & $\mathrm{C} 23 \mathrm{~B}-\mathrm{C} 22 \mathrm{~B}-\mathrm{C} 21 \mathrm{~B}$ & $121.2(2)$ \\
\hline $\mathrm{C} 23 \mathrm{~A}-\mathrm{C} 22 \mathrm{~A}-\mathrm{H} 22 \mathrm{~A}$ & 120.4 & $\mathrm{C} 23 \mathrm{~B}-\mathrm{C} 22 \mathrm{~B}-\mathrm{H} 22 \mathrm{~B}$ & 119.4 \\
\hline $\mathrm{C} 21 \mathrm{~A}-\mathrm{C} 22 \mathrm{~A}-\mathrm{H} 22 \mathrm{~A}$ & 120.4 & $\mathrm{C} 21 \mathrm{~B}-\mathrm{C} 22 \mathrm{~B}-\mathrm{H} 22 \mathrm{~B}$ & 119.4 \\
\hline $\mathrm{C} 24 \mathrm{~A}-\mathrm{C} 23 \mathrm{~A}-\mathrm{C} 22 \mathrm{~A}$ & $120.3(4)$ & $\mathrm{C} 22 \mathrm{~B}-\mathrm{C} 23 \mathrm{~B}-\mathrm{C} 24 \mathrm{~B}$ & $119.1(2)$ \\
\hline $\mathrm{C} 24 \mathrm{~A}-\mathrm{C} 23 \mathrm{~A}-\mathrm{H} 23 \mathrm{~A}$ & 119.8 & $\mathrm{C} 22 \mathrm{~B}-\mathrm{C} 23 \mathrm{~B}-\mathrm{H} 23 \mathrm{~B}$ & 120.4 \\
\hline $\mathrm{C} 22 \mathrm{~A}-\mathrm{C} 23 \mathrm{~A}-\mathrm{H} 23 \mathrm{~A}$ & 119.8 & $\mathrm{C} 24 \mathrm{~B}-\mathrm{C} 23 \mathrm{~B}-\mathrm{H} 23 \mathrm{~B}$ & 120.4 \\
\hline $\mathrm{C} 23 \mathrm{~A}-\mathrm{C} 24 \mathrm{~A}-\mathrm{C} 25 \mathrm{~A}$ & $120.3(4)$ & $\mathrm{C} 23 \mathrm{~B}-\mathrm{C} 24 \mathrm{~B}-\mathrm{C} 25 \mathrm{~B}$ & $120.1(2)$ \\
\hline $\mathrm{C} 23 \mathrm{~A}-\mathrm{C} 24 \mathrm{~A}-\mathrm{H} 24$ & 119.8 & $\mathrm{C} 23 \mathrm{~B}-\mathrm{C} 24 \mathrm{~B}-\mathrm{H} 24 \mathrm{~B}$ & 120.0 \\
\hline $\mathrm{C} 25 \mathrm{~A}-\mathrm{C} 24 \mathrm{~A}-\mathrm{H} 24$ & 119.8 & $\mathrm{C} 25 \mathrm{~B}-\mathrm{C} 24 \mathrm{~B}-\mathrm{H} 24 \mathrm{~B}$ & 120.0 \\
\hline $\mathrm{C} 24 \mathrm{~A}-\mathrm{C} 25 \mathrm{~A}-\mathrm{C} 20 \mathrm{~A}$ & $122.4(3)$ & $\mathrm{C} 20 \mathrm{~B}-\mathrm{C} 25 \mathrm{~B}-\mathrm{C} 24 \mathrm{~B}$ & $122.4(2)$ \\
\hline $\mathrm{C} 24 \mathrm{~A}-\mathrm{C} 25 \mathrm{~A}-\mathrm{H} 25 \mathrm{~A}$ & 118.8 & $\mathrm{C} 20 \mathrm{~B}-\mathrm{C} 25 \mathrm{~B}-\mathrm{H} 25 \mathrm{~B}$ & 118.8 \\
\hline $\mathrm{C} 20 \mathrm{~A}-\mathrm{C} 25 \mathrm{~A}-\mathrm{H} 25 \mathrm{~A}$ & 118.8 & $\mathrm{C} 24 \mathrm{~B}-\mathrm{C} 25 \mathrm{~B}-\mathrm{H} 25 \mathrm{~B}$ & 118.8 \\
\hline $\mathrm{C} 9 \mathrm{~A}-\mathrm{N} 1 \mathrm{~A}-\mathrm{N} 2 \mathrm{~A}-\mathrm{C} 12 \mathrm{~A}$ & $2.5(2)$ & $\mathrm{C} 9 \mathrm{~B}-\mathrm{N} 1 \mathrm{~B}-\mathrm{N} 2 \mathrm{~B}-\mathrm{C} 12 \mathrm{~B}$ & $0.1(2)$ \\
\hline $\mathrm{C} 8 \mathrm{~A}-\mathrm{N} 1 \mathrm{~A}-\mathrm{N} 2 \mathrm{~A}-\mathrm{C} 12 \mathrm{~A}$ & $-175.39(15)$ & $\mathrm{C} 8 \mathrm{~B}-\mathrm{N} 1 \mathrm{~B}-\mathrm{N} 2 \mathrm{~B}-\mathrm{C} 12 \mathrm{~B}$ & $179.13(14)$ \\
\hline $\mathrm{F} 1 \mathrm{~A}-\mathrm{C} 1 \mathrm{~A}-\mathrm{C} 2 \mathrm{~A}-\mathrm{C} 3 \mathrm{~A}$ & $-178.7(2)$ & $\mathrm{F} 1 \mathrm{~B}-\mathrm{C} 1 \mathrm{~B}-\mathrm{C} 2 \mathrm{~B}-\mathrm{C} 3 \mathrm{~B}$ & $-179.03(18)$ \\
\hline $\mathrm{C} 6 \mathrm{~A}-\mathrm{C} 1 \mathrm{~A}-\mathrm{C} 2 \mathrm{~A}-\mathrm{C} 3 \mathrm{~A}$ & $0.4(4)$ & $\mathrm{C} 6 \mathrm{~B}-\mathrm{C} 1 \mathrm{~B}-\mathrm{C} 2 \mathrm{~B}-\mathrm{C} 3 \mathrm{~B}$ & $1.1(3)$ \\
\hline $\mathrm{C} 1 \mathrm{~A}-\mathrm{C} 2 \mathrm{~A}-\mathrm{C} 3 \mathrm{~A}-\mathrm{C} 4 \mathrm{~A}$ & $0.9(4)$ & $\mathrm{C} 1 \mathrm{~B}-\mathrm{C} 2 \mathrm{~B}-\mathrm{C} 3 \mathrm{~B}-\mathrm{C} 4 \mathrm{~B}$ & $0.3(3)$ \\
\hline $\mathrm{C} 2 \mathrm{~A}-\mathrm{C} 3 \mathrm{~A}-\mathrm{C} 4 \mathrm{~A}-\mathrm{C} 5 \mathrm{~A}$ & $-1.4(3)$ & $\mathrm{C} 2 \mathrm{~B}-\mathrm{C} 3 \mathrm{~B}-\mathrm{C} 4 \mathrm{~B}-\mathrm{C} 5 \mathrm{~B}$ & $-1.0(3)$ \\
\hline $\mathrm{C} 2 \mathrm{~A}-\mathrm{C} 3 \mathrm{~A}-\mathrm{C} 4 \mathrm{~A}-\mathrm{C} 7 \mathrm{~A}$ & $176.6(2)$ & $\mathrm{C} 2 \mathrm{~B}-\mathrm{C} 3 \mathrm{~B}-\mathrm{C} 4 \mathrm{~B}-\mathrm{C} 7 \mathrm{~B}$ & $177.23(17)$ \\
\hline $\mathrm{C} 3 \mathrm{~A}-\mathrm{C} 4 \mathrm{~A}-\mathrm{C} 5 \mathrm{~A}-\mathrm{C} 6 \mathrm{~A}$ & $0.6(3)$ & $\mathrm{C} 3 \mathrm{~B}-\mathrm{C} 4 \mathrm{~B}-\mathrm{C} 5 \mathrm{~B}-\mathrm{C} 6 \mathrm{~B}$ & $0.3(3)$ \\
\hline $\mathrm{C} 7 \mathrm{~A}-\mathrm{C} 4 \mathrm{~A}-\mathrm{C} 5 \mathrm{~A}-\mathrm{C} 6 \mathrm{~A}$ & $-177.3(2)$ & $\mathrm{C} 7 \mathrm{~B}-\mathrm{C} 4 \mathrm{~B}-\mathrm{C} 5 \mathrm{~B}-\mathrm{C} 6 \mathrm{~B}$ & $-177.79(16)$ \\
\hline $\mathrm{F} 1 \mathrm{~A}-\mathrm{C} 1 \mathrm{~A}-\mathrm{C} 6 \mathrm{~A}-\mathrm{C} 5 \mathrm{~A}$ & $177.9(2)$ & $\mathrm{F} 1 \mathrm{~B}-\mathrm{C} 1 \mathrm{~B}-\mathrm{C} 6 \mathrm{~B}-\mathrm{C} 5 \mathrm{~B}$ & $178.42(17)$ \\
\hline $\mathrm{C} 2 \mathrm{~A}-\mathrm{C} 1 \mathrm{~A}-\mathrm{C} 6 \mathrm{~A}-\mathrm{C} 5 \mathrm{~A}$ & $-1.1(4)$ & $\mathrm{C} 2 \mathrm{~B}-\mathrm{C} 1 \mathrm{~B}-\mathrm{C} 6 \mathrm{~B}-\mathrm{C} 5 \mathrm{~B}$ & $-1.7(3)$ \\
\hline $\mathrm{C} 4 \mathrm{~A}-\mathrm{C} 5 \mathrm{~A}-\mathrm{C} 6 \mathrm{~A}-\mathrm{C} 1 \mathrm{~A}$ & $0.6(4)$ & $\mathrm{C} 4 \mathrm{~B}-\mathrm{C} 5 \mathrm{~B}-\mathrm{C} 6 \mathrm{~B}-\mathrm{C} 1 \mathrm{~B}$ & $0.9(3)$ \\
\hline $\mathrm{C} 5 \mathrm{~A}-\mathrm{C} 4 \mathrm{~A}-\mathrm{C} 7 \mathrm{~A}-\mathrm{O} 1 \mathrm{~A}$ & $-175.73(19)$ & $\mathrm{C} 5 \mathrm{~B}-\mathrm{C} 4 \mathrm{~B}-\mathrm{C} 7 \mathrm{~B}-\mathrm{O} 1 \mathrm{~B}$ & $-174.93(16)$ \\
\hline $\mathrm{C} 3 \mathrm{~A}-\mathrm{C} 4 \mathrm{~A}-\mathrm{C} 7 \mathrm{~A}-\mathrm{O} 1 \mathrm{~A}$ & $6.4(3)$ & $\mathrm{C} 3 \mathrm{~B}-\mathrm{C} 4 \mathrm{~B}-\mathrm{C} 7 \mathrm{~B}-\mathrm{O} 1 \mathrm{~B}$ & $7.0(2)$ \\
\hline $\mathrm{C} 5 \mathrm{~A}-\mathrm{C} 4 \mathrm{~A}-\mathrm{C} 7 \mathrm{~A}-\mathrm{C} 8 \mathrm{~A}$ & $6.3(3)$ & $\mathrm{C} 5 \mathrm{~B}-\mathrm{C} 4 \mathrm{~B}-\mathrm{C} 7 \mathrm{~B}-\mathrm{C} 8 \mathrm{~B}$ & $6.8(2)$ \\
\hline $\mathrm{C} 3 \mathrm{~A}-\mathrm{C} 4 \mathrm{~A}-\mathrm{C} 7 \mathrm{~A}-\mathrm{C} 8 \mathrm{~A}$ & $-171.63(18)$ & $\mathrm{C} 3 \mathrm{~B}-\mathrm{C} 4 \mathrm{~B}-\mathrm{C} 7 \mathrm{~B}-\mathrm{C} 8 \mathrm{~B}$ & $-171.34(15)$ \\
\hline
\end{tabular}




$\begin{array}{ll}\text { N2A-N1A-C8A-C7A } & -102.78(18) \\ \text { C9A-N1A-C8A-C7A } & 79.2(2) \\ \text { O1A-C7A-C8A-N1A } & 2.1(3) \\ \text { C4A-C7A-C8A-N1A } & -179.88(15) \\ \text { N2A-N1A-C9A-O2A } & 176.25(16) \\ \text { C8A-N1A-C9A-O2A } & -5.9(3) \\ \text { N2A-N1A-C9A-C10A } & -6.1(3) \\ \text { C8A-N1A-C9A-C10A } & 171.72(15) \\ \text { O2A-C9A-C10A-C11A } & -176.77(17) \\ \text { N1A-C9A-C10A-C11A } & 5.7(2) \\ \text { O2A-C9A-C10A-C19A } & 3.9(3) \\ \text { N1A-C9A-C10A-C19A } & -173.59(16) \\ \text { C9A-C10A-C11A-C12A } & -2.4(3) \\ \text { C19A-C10A-C11A-C12A } & 176.84(17) \\ \text { N1A-N2A-C12A-C11A } & 1.6(2) \\ \text { N1A-N2A-C12A-C13A } & 177.97(13) \\ \text { C10A-C11A-C12A-N2A } & -1.5(3) \\ \text { C10A-C11A-C12A-C13A } & -177.60(15) \\ \text { N2A-C12A-C13A-C18A } & -179.53(16) \\ \text { C11A-C12A-C13A-C18A } & -3.3(3) \\ \text { N2A-C12A-C13A-C14A } & -1.6(2) \\ \text { C11A-C12A-C13A-C14A } & 174.69(17) \\ \text { C18A-C13A-C14A-C15A } & -0.1(3) \\ \text { C12A-C13A-C14A-C15A } & -178.21(17) \\ \text { C13A-C14A-C15A-C16A } & 0.3(3) \\ \text { C14A-C15A-C16A-C17A } & -0.1(3) \\ \text { C15A-C16A-C17A-C18A } & -0.4(3) \\ \text { C16A-C17A-C18A-C13A } & 0.6(3) \\ \text { C14A-C13A-C18A-C17A } & -0.3(3) \\ \text { C12A-C13A-C18A-C17A } & 177.72(17) \\ \text { C11A-C10A-C19A-C20A } & -24.3(3) \\ \text { C9A-C10A-C19A-C20A } & 154.93(18) \\ \text { C10A-C19A-C20A-C21A } & 104.7(2) \\ \text { C10A-C19A-C20A-C25A } & -76.8(2) \\ \text { C25A-C20A-C21A-C22A } & 0.4(3) \\ \text { C19A-C20A-C21A-C22A } & 178.9(2) \\ \text { C20A-C21A-C22A-C23A } & 0.0(4) \\ \text { C21A-C22A-C23A-C24A } & 0.4(5) \\ \text { C22A-C23A-C24A-C25A } & -1.1(5) \\ \text { C23A-C24A-C25A-C20A } & 1.5(5) \\ \text { C21A-C20A-C25A-C24A } & -1.1(4) \\ \text { C19A-C20A-C25A-C24A } & -179.7(2) \\ & \end{array}$

\begin{tabular}{|c|c|}
\hline $\mathrm{N} 2 \mathrm{~B}-\mathrm{N} 1 \mathrm{~B}-\mathrm{C} 8 \mathrm{~B}-\mathrm{C} 7 \mathrm{~B}$ & $-99.93(16)$ \\
\hline $\mathrm{C} 9 \mathrm{~B}-\mathrm{N} 1 \mathrm{~B}-\mathrm{C} 8 \mathrm{~B}-\mathrm{C} 7 \mathrm{~B}$ & $79.18(19)$ \\
\hline $\mathrm{O} 1 \mathrm{~B}-\mathrm{C} 7 \mathrm{~B}-\mathrm{C} 8 \mathrm{~B}-\mathrm{N} 1 \mathrm{~B}$ & $-1.2(2)$ \\
\hline $\mathrm{C} 4 \mathrm{~B}-\mathrm{C} 7 \mathrm{~B}-\mathrm{C} 8 \mathrm{~B}-\mathrm{N} 1 \mathrm{~B}$ & $177.16(13)$ \\
\hline $\mathrm{N} 2 \mathrm{~B}-\mathrm{N} 1 \mathrm{~B}-\mathrm{C} 9 \mathrm{~B}-\mathrm{O} 2 \mathrm{~B}$ & $-179.01(16)$ \\
\hline $\mathrm{C} 8 \mathrm{~B}-\mathrm{N} 1 \mathrm{~B}-\mathrm{C} 9 \mathrm{~B}-\mathrm{O} 2 \mathrm{~B}$ & $2.0(2)$ \\
\hline $\mathrm{N} 2 \mathrm{~B}-\mathrm{N} 1 \mathrm{~B}-\mathrm{C} 9 \mathrm{~B}-\mathrm{C} 10 \mathrm{~B}$ & $0.2(2)$ \\
\hline $\mathrm{C} 8 \mathrm{~B}-\mathrm{N} 1 \mathrm{~B}-\mathrm{C} 9 \mathrm{~B}-\mathrm{C} 10 \mathrm{~B}$ & $-178.76(14)$ \\
\hline $\mathrm{O} 2 \mathrm{~B}-\mathrm{C} 9 \mathrm{~B}-\mathrm{C} 10 \mathrm{~B}-\mathrm{C} 11 \mathrm{~B}$ & $179.82(17)$ \\
\hline $\mathrm{N} 1 \mathrm{~B}-\mathrm{C} 9 \mathrm{~B}-\mathrm{C} 10 \mathrm{~B}-\mathrm{C} 11 \mathrm{~B}$ & $0.6(2)$ \\
\hline $\mathrm{O} 2 \mathrm{~B}-\mathrm{C} 9 \mathrm{~B}-\mathrm{C} 10 \mathrm{~B}-\mathrm{C} 19 \mathrm{~B}$ & $0.1(3)$ \\
\hline $\mathrm{N} 1 \mathrm{~B}-\mathrm{C} 9 \mathrm{~B}-\mathrm{C} 10 \mathrm{~B}-\mathrm{C} 19 \mathrm{~B}$ & $-179.12(14)$ \\
\hline $\mathrm{C} 9 \mathrm{~B}-\mathrm{C} 10 \mathrm{~B}-\mathrm{C} 11 \mathrm{~B}-\mathrm{C} 12 \mathrm{~B}$ & $-1.8(2)$ \\
\hline $\mathrm{C} 19 \mathrm{~B}-\mathrm{C} 10 \mathrm{~B}-\mathrm{C} 11 \mathrm{~B}-\mathrm{C} 12 \mathrm{~B}$ & $177.96(15)$ \\
\hline $\mathrm{N} 1 \mathrm{~B}-\mathrm{N} 2 \mathrm{~B}-\mathrm{C} 12 \mathrm{~B}-\mathrm{C} 11 \mathrm{~B}$ & $-1.2(2)$ \\
\hline $\mathrm{N} 1 \mathrm{~B}-\mathrm{N} 2 \mathrm{~B}-\mathrm{C} 12 \mathrm{~B}-\mathrm{C} 13 \mathrm{~B}$ & $175.68(13)$ \\
\hline $\mathrm{C} 10 \mathrm{~B}-\mathrm{C} 11 \mathrm{~B}-\mathrm{C} 12 \mathrm{~B}-\mathrm{N} 2 \mathrm{~B}$ & $2.2(2)$ \\
\hline $\mathrm{C} 10 \mathrm{~B}-\mathrm{C} 11 \mathrm{~B}-\mathrm{C} 12 \mathrm{~B}-\mathrm{C} 13 \mathrm{~B}$ & $-174.52(15)$ \\
\hline $\mathrm{N} 2 \mathrm{~B}-\mathrm{C} 12 \mathrm{~B}-\mathrm{C} 13 \mathrm{~B}-\mathrm{C} 18 \mathrm{~B}$ & $-168.90(15)$ \\
\hline $\mathrm{C} 11 \mathrm{~B}-\mathrm{C} 12 \mathrm{~B}-\mathrm{C} 13 \mathrm{~B}-\mathrm{C} 18 \mathrm{~B}$ & $8.0(2)$ \\
\hline $\mathrm{N} 2 \mathrm{~B}-\mathrm{C} 12 \mathrm{~B}-\mathrm{C} 13 \mathrm{~B}-\mathrm{C} 14 \mathrm{~B}$ & $8.0(2)$ \\
\hline $\mathrm{C} 11 \mathrm{~B}-\mathrm{C} 12 \mathrm{~B}-\mathrm{C} 13 \mathrm{~B}-\mathrm{C} 14 \mathrm{~B}$ & $-175.11(16)$ \\
\hline $\mathrm{C} 18 \mathrm{~B}-\mathrm{C} 13 \mathrm{~B}-\mathrm{C} 14 \mathrm{~B}-\mathrm{C} 15 \mathrm{~B}$ & $0.1(3)$ \\
\hline $\mathrm{C} 12 \mathrm{~B}-\mathrm{C} 13 \mathrm{~B}-\mathrm{C} 14 \mathrm{~B}-\mathrm{C} 15 \mathrm{~B}$ & $-176.92(16)$ \\
\hline $\mathrm{C} 13 \mathrm{~B}-\mathrm{C} 14 \mathrm{~B}-\mathrm{C} 15 \mathrm{~B}-\mathrm{C} 16 \mathrm{~B}$ & $-0.1(3)$ \\
\hline $\mathrm{C} 14 \mathrm{~B}-\mathrm{C} 15 \mathrm{~B}-\mathrm{C} 16 \mathrm{~B}-\mathrm{C} 17 \mathrm{~B}$ & $0.1(3)$ \\
\hline $\mathrm{C} 15 \mathrm{~B}-\mathrm{C} 16 \mathrm{~B}-\mathrm{C} 17 \mathrm{~B}-\mathrm{C} 18 \mathrm{~B}$ & $-0.2(3)$ \\
\hline $\mathrm{C} 16 \mathrm{~B}-\mathrm{C} 17 \mathrm{~B}-\mathrm{C} 18 \mathrm{~B}-\mathrm{C} 13 \mathrm{~B}$ & $0.2(3)$ \\
\hline $\mathrm{C} 14 \mathrm{~B}-\mathrm{C} 13 \mathrm{~B}-\mathrm{C} 18 \mathrm{~B}-\mathrm{C} 17 \mathrm{~B}$ & $-0.2(3)$ \\
\hline $\mathrm{C} 12 \mathrm{~B}-\mathrm{C} 13 \mathrm{~B}-\mathrm{C} 18 \mathrm{~B}-\mathrm{C} 17 \mathrm{~B}$ & $176.83(16)$ \\
\hline $\mathrm{C} 11 \mathrm{~B}-\mathrm{C} 10 \mathrm{~B}-\mathrm{C} 19 \mathrm{~B}-\mathrm{C} 20 \mathrm{~B}$ & $-78.2(2)$ \\
\hline $\mathrm{C} 9 \mathrm{~B}-\mathrm{C} 10 \mathrm{~B}-\mathrm{C} 19 \mathrm{~B}-\mathrm{C} 20 \mathrm{~B}$ & $101.59(18)$ \\
\hline $\mathrm{C} 10 \mathrm{~B}-\mathrm{C} 19 \mathrm{~B}-\mathrm{C} 20 \mathrm{~B}-\mathrm{C} 21 \mathrm{~B}$ & $132.6(2)$ \\
\hline $\mathrm{C} 10 \mathrm{~B}-\mathrm{C} 19 \mathrm{~B}-\mathrm{C} 20 \mathrm{~B}-\mathrm{C} 25 \mathrm{~B}$ & $-47.9(3)$ \\
\hline $\mathrm{C} 25 \mathrm{~B}-\mathrm{C} 20 \mathrm{~B}-\mathrm{C} 21 \mathrm{~B}-\mathrm{C} 22 \mathrm{~B}$ & $1.1(4)$ \\
\hline $\mathrm{C} 19 \mathrm{~B}-\mathrm{C} 20 \mathrm{~B}-\mathrm{C} 21 \mathrm{~B}-\mathrm{C} 22 \mathrm{~B}$ & $-179.4(2)$ \\
\hline $\mathrm{C} 20 \mathrm{~B}-\mathrm{C} 21 \mathrm{~B}-\mathrm{C} 22 \mathrm{~B}-\mathrm{C} 23 \mathrm{~B}$ & $-1.2(4)$ \\
\hline $\mathrm{C} 21 \mathrm{~B}-\mathrm{C} 22 \mathrm{~B}-\mathrm{C} 23 \mathrm{~B}-\mathrm{C} 24 \mathrm{~B}$ & $0.8(4)$ \\
\hline $\mathrm{C} 22 \mathrm{~B}-\mathrm{C} 23 \mathrm{~B}-\mathrm{C} 24 \mathrm{~B}-\mathrm{C} 25 \mathrm{~B}$ & $-0.4(4)$ \\
\hline $\mathrm{C} 21 \mathrm{~B}-\mathrm{C} 20 \mathrm{~B}-\mathrm{C} 25 \mathrm{~B}-\mathrm{C} 24 \mathrm{~B}$ & $-0.7(4)$ \\
\hline $\mathrm{C} 19 \mathrm{~B}-\mathrm{C} 20 \mathrm{~B}-\mathrm{C} 25 \mathrm{~B}-\mathrm{C} 24 \mathrm{~B}$ & $179.8(2)$ \\
\hline $\mathrm{C} 23 \mathrm{~B}-\mathrm{C} 24 \mathrm{~B}-\mathrm{C} 25 \mathrm{~B}-\mathrm{C} 20 \mathrm{~B}$ & $0.4(5)$ \\
\hline
\end{tabular}

Hydrogen-bond geometry $\left(A,{ }^{\circ}\right)$

$\mathrm{Cg} 1$ is the centroid of the $\mathrm{N} 1 A / \mathrm{N} 2 A / \mathrm{C} 9 A-\mathrm{C} 12 A$ ring.

\begin{tabular}{lllll}
\hline$D-\mathrm{H} \cdots A$ & $D-\mathrm{H}$ & $\mathrm{H} \cdots A$ & $D \cdots A$ & $D-\mathrm{H} \cdots A$ \\
\hline $\mathrm{C} 15 A-\mathrm{H} 15 A \cdots \mathrm{F} 1 A^{\mathrm{i}}$ & 0.93 & 2.49 & $3.263(3)$ & 141
\end{tabular}




$\begin{array}{lllll}\mathrm{C} 15 B-\mathrm{H} 15 B \cdots \mathrm{F} 1 B^{\mathrm{ii}} & 0.93 & 2.56 & 3.310(3) & 138 \\ \mathrm{C} 8 A-\mathrm{H} 8 B \cdots \mathrm{O} 1 A^{\mathrm{iii}} & 0.97 & 2.50 & 3.466(3) & 179 \\ \mathrm{C} 8 B-\mathrm{H} 8 D \cdots \mathrm{O} 1 B^{\mathrm{iv}} & 0.97 & 2.49 & 3.458(2) & 176 \\ \mathrm{C} 19 A-\mathrm{H} 19 A \cdots C g 1^{\text {iv }} & 0.97 & 2.93 & 3.845(2) & 158\end{array}$

Symmetry codes: (i) $-x+2,-y+1,-z$; (ii) $-x-1,-y,-z+1$; (iii) $x+1, y, z$; (iv) $x-1, y, z$. 